Modelagem de epidemias via sistemas de partículas interagentes

\author{
Valdivino Vargas Júnior
}

TESE APRESENTADA

\author{
Instituto DE MATEMÁtica E EstatísticA \\ Universidade DE SÃo PAUlo \\ PARA \\ OBTENÇẪO DO TÍTULO \\ DOUTOR EM CIÊNCIAS
}

Programa: Estatística

Orientador: Prof. Dr. Fábio Prates Machado

Durante o desenvolvimento deste trabalho o autor recebeu auxílio

financeiro do $\mathrm{CNPq}$

São Paulo, maio de 2010 


\section{Modelagem de epidemias via sistemas de partículas interagentes}

\footnotetext{
Este exemplar corresponde à redação final da tese devidamente corrigida e defendida por Valdivino Vargas Júnior e aprovada pela Comissão Julgadora.
}

Banca Examinadora:

- Prof. Dr. Fábio Prates Machado-(Presidente)-IME-USP.

- Prof. Dr. Nome Élcio Lebensztayn - IME-USP.

- Prof. Dr. Fernando Pigeard de Almeida Prado - FFCLRPUSP

- Prof. Dr. Marcelo Sobottka - UFSC.

- Prof. Dr. Maurício Zuluaga Martinez - UFPE. 


\section{Agradecimentos}

Sinceros agradecimentos a todos aqueles que de alguma forma contribuíram para a confecção desta tese. Em especial, ao meu orientador Fábio Prates Machado pela dedicada orientação e apoio durante todo o desenvolvimento do trabalho. E também a minha família, destacando principalmente a minha esposa Graciele, pessoa que sempre me apoiou e me incentivou durante esta caminhada. A Deus pela vida e aos amigos pela confiança e incentivo. 


\section{Resumo}

Estudamos um sistema de partículas a tempo discreto cuja dinâmica é a seguinte. Considere que no instante inicial sobre cada inteiro não negativo há uma partícula, inicialmente inativa. A partícula da origem é ativada e instantaneamente ativa um conjunto aleatório contíguo de partículas que estão a sua direita. Como regra, no instante seguinte ao que foi ativada, cada partícula ativa realiza esta mesma dinâmica de modo independentemente de todo o resto. Dizemos que o processo sobrevive se em qualquer momento sempre há ao menos uma partícula ativa. Chamamos este processo de Firework, associando a dinâmica de ativação de uma partícula inativa a uma infecção ou explosão. Nosso interesse é estabelecer se o processo tem probabilidade positiva de sobrevivência e apresentar limites para esta probabilidade. Isto deve ser feito em função da distribuição da variável aleatória que define o raio de ação de uma partícula. Associando o processo de ativação a uma infecção, podemos pensar este modelo como um modelo epidêmico.

Consideramos também algumas variações dessa dinâmica. Dentre elas, variantes com partículas distribuídas sobre a semirreta dos reais positivos (nesta vertente, existem condições para as distâncias entre partículas consecutivas) e também com as partículas distribuídas sobre vértices de árvores. Estudamos também para esses casos a transição de fase e probabilidade de sobrevivência. Nesta variante os resultados obtidos são funções da sequência de distribuições dos alcances das explosões e da estrutura dos lugares onde se localizam as partículas. Consideramos também variações do modelo onde cada partícula ao ser ativada, permanece ativa durante um tempo aleatório e nesse período emite explosões que ocorrem em instantes aleatórios.

Palavras-chave: modelo epidêmico, processo de ramificação, transição de fase. 


\section{Abstract}

We studied a discrete time particle system whose dynamic is as follows. Consider that at time zero, on each non-negative integer, there is a particle, initially inactive. A particle which is placed at origin is activated and instantly activates a contiguous random set of particles that is on its right. As a rule, the next moment to what it has been activated, each active particle carries the same behavior independently of the rest. We say that the process survives if the amount of particles activated along the process is infinite. We call this the Firework process, associating the activation dynamic of a particle to an infection or explosion process. Our interest is to establish whether the process has positive probability of survival and to present limits to this probability. This is done according to the distribution random variable that defines the radius of infection of each active particle, Associating the activation process to an infection, we think this model as a model epidemic.

We also consider some variations of this dynamic. Among them, variants with particles distributed over the half line (there are conditions for the distances between consecutive particles) and also with particles distributed over the vertices of a tree. We studied phase transitions and the correspondent survival probability. In this variant the results depend on the sequence of probability distributions for the range of the explosions and on the particles displacement. We also consider a variation where each particle after activated, remains active during a random time period emitting explosions that occur in random moments. Keywords: epidemic model, branching process, phase transition. 


\section{Sumário}

1 Introdução 1

1.1 Considerações Iniciais $\ldots \ldots \ldots \ldots$

1.2 Motivação . . . . . . . . . . . . . . . . . . . 1

1.3 Organização do Trabalho . . . . . . . . . . . . . . 2

2 Processo Firework Discreto Unidimensional 5

2.1 Condições Gerais de Sobrevivência . . . . . . . . . . . . 5

2.2 O caso Homogêneo . . . . . . . . . . . . . . . . . . 10

2.3 O caso Heterogêneo . . . . . . . . . . . . . . . . . 15

2.4 Processo Firework Discreto Unidimensional Reverso . . . . . 19

2.4.1 O caso Reverso Homogêneo . . . . . . . . . . . . . 20

2.4.2 O caso Reverso Heterogêneo . . . . . . . . . . . . 25

3 Processo Firework Contínuo Unidimensional 27

3.1 Resultados preliminares . . . . . . . . . . . . . . 28

3.2 O caso Homogêneo . . . . . . . . . . . . . . . . . . 31

3.3 O caso Heterogêneo $\ldots \ldots \ldots \ldots$

4 Processo Firework Discreto em Árvores $\quad 37$

4.1 Árvore Homogênea . . . . . . . . . . . . . . . . . . . 38

4.1.1 Condições gerais para sobrevivência . . . . . . . . 38

4.1.2 Limites para a Probabilidade de Sobrevivência . . . . 43

4.2 Árvore k-periódica . . . . . . . . . . . . . . . . . . . . . 49

4.2.1 Condições gerais para sobrevivência . . . . . . . . 52

4.2.2 Limites para a Probabilidade de Sobrevivência . . . . 55

4.3 Árvore Esfericamente Simétrica . . . . . . . . . . . . . . . . 61

5 Apêndice $\quad 65$

5.1 Resultados básicos . . . . . . . . . . . . . . 65

5.2 Generalização do Lema de Borel-Cantelli . . . . . . . . . . . . 66 
5.3 Processos de Galton-Watson . . . . . . . . . . . . . 67

5.4 Processos de Galton-Watson em Meios Aleatórios . . . . . . . 69 


\section{Capítulo 1}

\section{Introdução}

\subsection{Considerações Iniciais}

Estudamos um sistema de partículas cuja dinâmica é a seguinte. Consideramos um conjunto infinito de partículas, todas inativas. No instante inicial, uma das partículas é ativada. Uma partícula ativa é capaz de emitir uma quantidade finita de explosões de alcance finito e aleatório. Uma partícula inativa se torna ativa ao ser atingida por uma explosão. Dizemos que o processo sobrevive, se para cada instante de tempo existe pelo menos uma partícula ativa.

\subsection{Motivação}

A difusão de uma infecção ou informação tem sido bastante estudada por diversos probabilistas. Nós consideramos um tipo particular de difusão onde todos os indivíduos do sistema são suscetíveis e existe uma certa probabilidade de que um indivíduo infectado transmita a infecção para todos os vizinhos em uma vizinhança aleatória dele. Em termos de percolação, estamos considerando um modelo de percolação dependente de longo alcance.

Neste trabalho, cada indivíduo é visto como uma partícula. Indivíduos suscetíveis são descritos como partículas inativas. Ser infectado está associado a idéia da partícula ser ativada. Dizemos que uma partícula ativa emite explosões de alcance finito e aleatório. Isto em termos de modelo epidêmico quer dizer que ao ser infectado, o indivíduo começa a emitir, digamos "vírus" infectando indivíduos em uma vizinhança finita e aleatória dele.

Podemos também pensar em um modelo de difusão de informação. Nesse 
caso, cada partícula pode ser vista como um potencial transmissor de informação. Assim, a idéia da partícula ser ativada é associada a idéia do potencial transmissor receber a informação. Este pode ser um indivíduo ou mesmo uma central capaz de transmitir dados. Nessa linha de pensamento, o transmissor ao receber a informação, a repassa para uma vizinhança finita e aleatória dele. Isto pode ser associado a idéia da partícula emitir explosões.

Nosso objetivo é estudar o comportamento desse modelo com relação à sobrevivência do processo em algumas situações distintas. Estudamos a questão da transição de fase e encontramos limites para a probabilidade de sobrevivência.

\subsection{Organização do Trabalho}

No capítulo 2, estudamos uma versão do processo a tempo discreto. Consideramos um conjunto de partículas sobre a reta metade. Estas se encontram em posições $u_{n}$, onde $n$ indica que a partícula é a n-ésima mais próxima da origem. Nos referimos a ela como partícula $n$. A partícula que dá início ao processo se encontra na posição $u_{0}=0$, isto é, sobre a origem. Esta, no instante $t=0$ é ativada e no instante seguinte emite uma única explosão de alcance aleatório e finito $R_{0}$. Partículas inativas que sejam atingidas por esta explosão se tornam ativas e realizam esta mesma dinâmica e assim por diante. Cada partícula é ativada uma única vez, isto é, se num dado instante uma partícula $n$ é atingida por alguma explosão, ela emite uma explosão de alcance $R_{n}$ e não volta a repetir isso, mesmo que seja novamente atingida por outra explosão. Os alcances de explosões dados pelas variáveis aleatórias $\left\{R_{n}\right\}_{n \in \mathbb{Z}_{+}}$são independentes. Chamamos esse processo de Firework Discreto. Apresentamos dois casos: homogêneo e heterogêneo. No caso homogêneo, as variáveis $\left\{R_{n}\right\}_{n \in \mathbb{Z}_{+}}$são variáveis aleatórias independentes e identicamente distribuídas (v.a.i.i.d.) e as partículas se encontram nas posições $u_{n}=n$. Para esse caso, apresentamos transição de fase. No caso heterogêneo, as variáveis $\left\{R_{n}\right\}_{n \in \mathbb{Z}_{+}}$são independentes, mas não são identicamente distribuídas e existem condições para a distância entre partículas consecutivas. Para esse caso, apresentamos condições suficientes para a existência e ausência de chance positiva de sobrevivência do processo. Tanto para o caso homogêneo como para o heterogêneo apresentamos um limite inferior para a probabilidade de sobrevivência do processo. Na última seção do capítulo apresentamos uma variante para o processo Firework Discreto a qual chamamos Firework Reverso. Nesta uma partícula $n$ só se torna ativa se estiver a uma distância $R_{n}$ de alguma partícula ativa. Inicialmente 
apenas a partícula 0 é ativada. Para esse modelo apresentamos os casos homogêneos e heterogêneos com as partículas distribuídas sobre os inteiros não-negativos. Fazemos também uma comparação entre o caso Reverso homogêneo e o Firework Discreto Homogêneo. Os dois modelos apresentam diferenças substanciais em suas condições de sobrevivência.

No capítulo 3, estudamos uma versão do processo a tempo contínuo. Consideramos partículas distribuídas sobre a semirreta dos reais positivos nas posições $u_{n}$, onde $n$ indica que a partícula é a n-ésima mais próxima da origem. Nos referimos a ela como partícula $n$. A partícula que dá início ao processo se encontra na posição $u_{0}=0$, isto é, sobre a origem. No instante $t=0$, todas as partículas estão inativas, exceto aquela na origem que é inicialmente ativada e dá início ao processo. Uma partícula $n$ ao ser ativada, assim permanece durante um tempo aleatório $T_{n}$. Durante este tempo a partícula $n$ emite explosões independentes com alcances aleatórios e finitos $\left\{\bar{R}_{n}^{j}\right\}_{j \in \mathbb{Z}_{+}^{*}}$ As explosões ocorrem em instantes pontuais aleatórios de modo que o tempo entre explosões consecutivas é exponencial com parâmetro $\mu_{n}$. Quando uma partícula inativa é atingida por uma explosão ela se torna ativa. Cada partícula somente pode ser ativada uma única vez, isto é, se uma partícula ativa tem seu tempo de ativação encerrado, ela não pode ser ativada novamente, mesmo que atingida pela explosão de outra partícula. Chamamos esse processo de Firework Contínuo. Consideramos dois casos: o homogêneo e o heterogêneo. No caso homogêneo os alcances de explosões são v.a.i.i.d.. Também são v.a.i.i.d. os tempos de ativação das partículas e os tempos entre explosões. No caso heterogêneo todas estas variáveis são independentes, entretanto pelo menos um dos conjuntos de variáveis é não identicamente distribuído ou as partículas não estão necessariamente sobre $\mathbb{Z}_{+}$. Para o caso heterogêneo, apresentamos condições suficientes para a existência e ausência de chance positiva de sobrevivência do modelo. Para o caso homogêneo apresentamos transição de fase. Tanto para o caso homogêneo como para o heterogêneo apresentamos um limite inferior para a probabilidade de sobrevivência do processo.

No capítulo 4, consideramos o processo Firework discreto sobre árvores. Nesse caso, cada vértice da árvore possui exatamente uma partícula. Inicialmente, todas as partículas estão inativas, exceto a partícula sobre a raiz. Esta emite uma explosão de alcance aleatório. Cada partícula atingida pela explosão, emite no próximo instante uma única explosão e assim por diante. Verificamos condições de sobrevivência em árvores homogêneas e periódicas. Apresentamos também limitantes para a probabilidade de sobrevivência nestas árvores. Finalizamos o capítulo apresentando uma condição suficiente para sobrevivência com probabilidade positiva em árvores esferi- 
camente simétricas. 


\section{Capítulo 2}

\section{Processo Firework Discreto Unidimensional}

A dinâmica do Processo Firework Discreto Unidimensional é a seguinte. Consideramos um conjunto de partículas sobre a semirreta dos reais positivos. Estas se encontram em posições $u_{n}$, onde $n$ indica que a partícula é a n-ésima mais próxima da origem. Nos referimos a ela como partícula $n$. A partícula que inicia o processo se encontra na posição $u_{0}=0$, isto é, sobre a origem. Esta, no instante $t=0$ é ativada e no instante seguinte emite uma única explosão de alcance aleatório e finito $R_{0}$. Partículas inativas que sejam atingidas por esta explosão realizam esta mesma dinâmica e assim por diante. Cada partícula é ativada uma única vez, isto é, se num dado instante uma partícula $n$ é atingida por alguma explosão, ela emite uma explosão de alcance $R_{n}$ e não volta a repetir isso, mesmo que seja novamente atingida por outra explosão. Os alcances de explosões dados pelas variáveis aleatórias $\left\{R_{n}\right\}_{n \in \mathbb{Z}_{+}}$são independentes.

\subsection{Condições Gerais de Sobrevivência}

Consideremos as seguintes definições:

Definição 2.1.0.1. Sejam $\left\{R_{n}\right\}_{n \in \mathbb{Z}_{+}}$variáveis aleatórias independentes assumindo valores nos reais não negativos, com distribuição $\left\{\mathbf{P}_{\mathbf{n}}\right\}_{n \in \mathbb{Z}_{+}}$respectivamente. Defina

$$
\mathbb{P}=\prod_{n=0}^{\infty} \mathbf{P}_{n}
$$


$\mathbb{P}$ é a medida de probabilidade para o Processo Firework.

Observação. $\mathbb{P}\left(R_{n} \leq k\right)=\mathbf{P}_{n}\left(R_{n} \leq k\right)$

Definição 2.1.0.2. Considere o evento $V_{n}$ : "Partícula $n$ é atingida"e o evento

$$
V:=\lim _{n \rightarrow \infty} V_{n}=\bigcap_{n=0}^{\infty} V_{n}
$$

A última igualdade segue do fato de que $V_{n+1} \subseteq V_{n}$. Note que o Processo Firework Discreto Unidimensional sobrevive se e somente se $V$ ocorre.

Proposição 2.1.0.1. Considere um Processo Firework Discreto Unidimensional onde as posições das partículas satisfazem $u_{n+1}-u_{n} \geq k$ e cujas marginais satisfaçam

i) $\mathbb{P}\left(R_{i}<k\right) \in(0,1)$ para todo $i$

ii) $\lim _{n \rightarrow \infty} \prod_{i=0}^{n-1} \mathbb{P}\left(R_{i}<k(2 n-i)\right)=1$

iii) $\lim _{n \rightarrow \infty} \prod_{i=n}^{2 n-1} \mathbb{P}\left(R_{i}<k(2 n-i)\right)>0$

Então o processo morre quase certamente.

Prova da Proposição 2.1.0.1

Para a prova faremos uma pequena modificação no modelo. Admitiremos que as partículas se encontram nas posições $\mathrm{u}_{n}=k n$. Observe que a não sobrevivência nesse modelo implica a não sobrevivência no modelo original. Sejam:

$$
A_{n}:=\bigcup_{i=0}^{n-1}\left\{R_{i} \geq k(2 n-i)\right\}
$$

e

$$
B_{n}:=\bigcup_{i=n}^{2 n-1}\left\{R_{i} \geq k(2 n-i)\right\}
$$

Observe que os eventos $B_{n}$ e $V_{n}$ são independentes. Além disso,

$$
V_{2 n} \subseteq V_{n} \cap\left[A_{n} \cup B_{n}\right]
$$

e $\log 0$

$$
\begin{aligned}
\mathbb{P}\left(V_{2 n}\right) & \leq \mathbb{P}\left(V_{n} \cap\left[A_{n} \cup B_{n}\right]\right)=\mathbb{P}\left(\left[V_{n} \cap A_{n}\right] \cup\left[V_{n} \cap B_{n}\right]\right) \leq \mathbb{P}\left(A_{n} \cup\left[V_{n} \cap B_{n}\right]\right) \\
& \leq \mathbb{P}\left(A_{n}\right)+\mathbb{P}\left(B_{n} \cap V_{n}\right)=\mathbb{P}\left(A_{n}\right)+\mathbb{P}\left(B_{n}\right) \mathbb{P}\left(V_{n}\right)
\end{aligned}
$$


Isto é,

$$
\mathbb{P}\left(V_{2 n}\right) \leq \mathbb{P}\left(A_{n}\right)+\mathbb{P}\left(B_{n}\right) \mathbb{P}\left(V_{n}\right)
$$

Lema 2.1.0.1. Nas hipóteses da Proposição 2.1.0.1

$$
\lim _{n \rightarrow \infty} \mathbb{P}\left(A_{n}\right)=0
$$

Prova do Lema 2.1.0.1

$$
\mathbb{P}\left(A_{n}\right)=\mathbb{P}\left(\bigcup_{i=0}^{n-1}\left\{R_{i} \geq k(2 n-i)\right\}\right)=1-\prod_{i=0}^{n-1} \mathbb{P}\left(R_{i}<k(2 n-i)\right) \rightarrow 0
$$

Lema 2.1.0.2. Nas hipóteses da Proposição 2.1.0.1 existe $\lambda \in(0,1)$ tal que para todo $n$

$$
\mathbb{P}\left(B_{n}\right) \leq \lambda
$$

Prova do Lema 2.1.0.2

$$
\begin{aligned}
\mathbb{P}\left(B_{n}\right) & =\mathbb{P}\left(\bigcup_{i=n}^{2 n-1}\left\{R_{i} \geq k(2 n-i)\right\}\right)=1-\mathbb{P}\left(\bigcap_{i=n}^{2 n-1}\left\{R_{i}<k(2 n-i)\right\}\right) \\
& =1-\prod_{i=n}^{2 n-1} \mathbb{P}\left(R_{i}<k(2 n-i)\right)
\end{aligned}
$$

Assim, o Lema 5.1.0.2 (ver apêndice) e a condição $i i i)$ nos garante que existe $\lambda \in(0,1)$ tal que

$$
\mathbb{P}\left(B_{n}\right) \leq \lambda
$$

para todo n. E isto conclui a prova do Lema 2.1.0.2.

Voltemos a prova da Proposição. De 2.1.4 e do Lema 2.1.0.2 segue

$$
\mathbb{P}\left(V_{2^{m+1}}\right) \leq \mathbb{P}\left(A_{2^{m}}\right)+\lambda \mathbb{P}\left(V_{2^{m}}\right)
$$

Tome $\epsilon>0$. Escolha $\epsilon_{0}$ e $\epsilon_{1}$ tais que

$$
\epsilon=\frac{\epsilon_{0}}{1-\lambda}+\epsilon_{1}
$$

Do Lema 2.1.0.1 existe $m_{0}$ tal que para todo $m>m_{0}, \mathbb{P}\left(A_{2^{m}}\right)<\epsilon_{0}$. Por outro lado, existe $m_{1}$ tal que para todo $m>m_{1}, \lambda^{m}<\epsilon_{1}$. Seja $M=$ 
$\max \left\{m_{0}, m_{1}\right\}$. Observe que para todo $m>m_{1}$ :

$\mathbb{P}\left(V_{2^{m+1}}\right)<\epsilon_{0}+\lambda \mathbb{P}\left(V_{2^{m}}\right)$

$\mathbb{P}\left(V_{2^{m+2}}\right)<\epsilon_{0}+\lambda \epsilon_{0}+\lambda^{2} \mathbb{P}\left(V_{2^{m}}\right)$

$\mathbb{P}\left(V_{4^{m}}\right)<\frac{\left(1-\lambda^{m}\right)}{1-\lambda} \epsilon_{0}+\lambda^{m} \mathbb{P}\left(V_{2^{m}}\right)$

Logo

$$
\mathbb{P}\left(V_{4^{m}}\right)<\frac{\epsilon_{0}}{1-\lambda}+\lambda^{m}
$$

Portanto, se $m>M$,

$$
\mathbb{P}\left(V_{4^{m}}\right)<\frac{\epsilon_{0}}{1-\lambda}+\epsilon_{1}=\epsilon
$$

Isto significa que,

$$
\lim _{m \rightarrow \infty} \mathbb{P}\left(V_{4^{m}}\right)=0
$$

Para finalizar, basta observar que $V_{n+1} \subset V_{n}$ e isto implica pela continuidade da probabilidade que $\mathbb{P}(V)=0$

Proposição 2.1.0.2. Considere um Processo Firework Discreto onde as posições das partículas satisfazem $\left|u_{n+1}-u_{n}\right| \leq m$. As condições

i) $\mathbb{P}\left(R_{i}<m\right) \in(0,1)$

ii) $\sum_{n=0}^{\infty} a_{n}<\infty$ onde $a_{n}=\prod_{j=0}^{n} \mathbb{P}\left(R_{n-j}<(j+1) m\right)$

são suficientes para que o processo sobreviva com probabilidade positiva. Nesse caso,

$$
\mathbb{P}(V) \geq \prod_{i=0}^{\infty}\left[1-\prod_{j=0}^{i} \mathbb{P}\left(R_{i-j}<(j+1)\right) m\right]
$$

Prova da Proposição 2.1.0.2

Para a prova faremos uma pequena modificação no modelo. Admitiremos que as partículas se encontram nas posições $u_{n}=m n$. Observe que a sobrevivência nesse modelo implica a sobrevivência no modelo original.

Antes de iniciar a prova da proposição, apresentaremos alguns lemas que serão úteis para a sua prova. 


\section{Lema 2.1.0.3.}

i) $V_{k+1}=V_{k} \bigcap\left\{\bigcup_{i=0}^{k}\left(R_{k-i} \geq(i+1) m\right)\right\}$.

ii) $V_{k}$ e $\bigcup_{i=0}^{k}\left(R_{k-i} \geq(i+1) m\right)$ são eventos crescentes.

iii) $\mathbb{P}\left(V_{n}\right)>0 \forall n$.

Prova do Lema 2.1.0.3

i) segue direto da definição do processo. Para $i i)$ sejam $\omega_{1}$ e $\omega_{2}$ dois pontos do espaço amostral. Diremos que $\omega_{1} \leq \omega_{2}$ se e somente se $R_{n}\left(\omega_{1}\right) \leq R_{n}\left(\omega_{2}\right)$ para todo $n$ inteiro não negativo. Com esta ordenação, os eventos em $i i)$ são crescentes. iii) segue da primeira hipótese da Proposição.

\section{Lema 2.1.0.4.}

$$
\mathbb{P}\left(V_{k} \bigcap\left\{\bigcup_{i=0}^{k}\left(R_{k-i} \geq(i+1) m\right)\right\}\right) \geq \mathbb{P}\left(V_{k}\right) \cdot \mathbb{P}\left(\bigcup_{i=0}^{k}\left(R_{k-i} \geq(i+1) m\right)\right)
$$

Prova do Lema 2.1.0.4

Segue do Lema 2.1.0.3 e da aplicação da desigualdade FKG (Lema 5.1.0.1).

\section{Lema 2.1.0.5.}

$$
\mathbb{P}\left(V_{k+n}\right) \geq \mathbb{P}\left(V_{k}\right) \cdot \prod_{i=0}^{n-1}\left[1-\prod_{j=0}^{k+i} \mathbb{P}\left(R_{k+i-j}<(j+1) m\right)\right]
$$

Prova do Lema 2.1.0.5

Pelo Lema 2.1.0.4 e devido a independência das variáveis $R_{j}$, segue que

$$
\begin{aligned}
\mathbb{P}\left(V_{k+1}\right) & \geq \mathbb{P}\left(V_{k}\right) \cdot \mathbb{P}\left(\bigcup_{j=0}^{k} R_{k-j} \geq(j+1) m\right) \\
& =\mathbb{P}\left(V_{k}\right) \cdot\left[1-\prod_{j=0}^{k} \mathbb{P}\left(R_{k-j}<(j+1) m\right)\right] \\
\mathbb{P}\left(V_{k+1}\right) & \geq \mathbb{P}\left(V_{k}\right) \cdot\left[1-\prod_{j=0}^{k} \mathbb{P}\left(R_{k-j}<(j+1) m\right)\right]
\end{aligned}
$$


Analogamente, obtemos:

$$
\mathbb{P}\left(V_{k+2}\right) \geq \mathbb{P}\left(V_{k+1}\right) \cdot\left[1-\prod_{j=0}^{k+1} \mathbb{P}\left(R_{k+1-j}<(j+1) m\right)\right]
$$

De (2.1.6) e (2.1.7) temos:

$$
\mathbb{P}\left(V_{k+2}\right) \geq \mathbb{P}\left(V_{k}\right) \cdot\left[1-\prod_{j=0}^{k} \mathbb{P}\left(R_{k-j}<(j+1) m\right)\right]\left[1-\prod_{j=0}^{k+1} \mathbb{P}\left(R_{k+1-j}<(j+1) m\right)\right]
$$

e por argumento indutivo concluímos a prova.

Tome $\mathrm{k}=0$ no Lema 2.1.0.5

$$
\mathbb{P}\left(V_{n}\right) \geq \mathbb{P}\left(V_{0}\right) \cdot \prod_{i=0}^{n-1}\left[1-\prod_{j=0}^{i} \mathbb{P}\left(R_{i-j}<(j+1) m\right)\right]
$$

Por definição temos $\mathbb{P}\left(V_{0}\right)=1$. Pela continuidade da probabilidade

$$
\mathbb{P}(V) \geq \prod_{i=0}^{\infty}\left[1-\prod_{j=0}^{i} \mathbb{P}\left(R_{i-j}<(j+1)\right) m\right] .
$$

Por outro lado pelo Lema 5.1.0.2 temos que

$$
\prod_{i=0}^{\infty}\left[1-\prod_{j=0}^{i} \mathbb{P}\left(R_{i-j}<(j+1) m\right)\right]>0 \Leftrightarrow \sum_{n=0}^{\infty} \prod_{j=0}^{n} \mathbb{P}\left(R_{n-j}<(j+1) m\right)<\infty
$$

com o que concluímos a prova

\subsection{O caso Homogêneo}

Para o caso homogêneo, temos $m=1, u_{n}=n$ e $R_{n}={ }^{d} R$ para todo $n$.

Teorema 2.2.1. Para o Processo Firework Discreto homogêneo onde

$$
\mathbb{P}(R<1) \in(0,1)
$$

considere a quantidade

$$
L=\lim _{n \rightarrow \infty} n \mathbb{P}(R \geq n)
$$


- Se $L>1$, então o processo homogêneo sobrevive com probabilidade positiva. Nesse caso

$$
\mathbb{P}(V) \geq \prod_{i=0}^{\infty}\left[1-\prod_{j=0}^{i} \mathbb{P}(R<j+1)\right]
$$

- Se $L<1$, então o processo homogêneo morre quase certamente;

- Se $L=1$ e existe um $N$ tal que para todo $n \geq N$

$$
\mathbb{P}(R \geq n)<\frac{1}{n-1},
$$

então o processo morre quase certamente.

Prova do Teorema 2.2.1

Pela Proposição 2.1.0.2 o Processo Firework Discreto tem probabilidade positiva de sobreviver se

$$
\begin{aligned}
& \text { i) } \mathbb{P}(R<1) \in(0,1) \\
& \text { ii) } \sum_{n=0}^{\infty} a_{n}<\infty \text { onde } a_{n}=\prod_{j=0}^{n} \mathbb{P}(R<j+1)
\end{aligned}
$$

Sob essas condições é possível via Lema 5.1.0.3 obter que o processo tem probabilidade positiva de sobreviver se

$$
\mathbb{P}(R<1) \in(0,1) \text { e } \lim _{n \rightarrow \infty} n \mathbb{P}(R \geq n)>1 .
$$

Agora vamos mostrar que as outras condições implicam em morte quase certa do processo.

Considere o seguinte processo auxiliar. Mesmo sem ativação, cada partícula de forma independente causa uma explosão, cujos alcances são definidos segundo variáveis aleatórias independentes e identicamente distribuídas com a mesma distribuição de uma variável aleatória $R$. Chamemos essa dinâmica de processo Firework sem ativação. Consideremos o evento $C$ : "A partir de um certo n, todas as partículas são atingidas pela explosão de alguma outra". Considere a realização conjunta dos dois processos. Note que se $\omega \in V$ então $\omega \in C$. Assim

$$
\mathbb{P}(V) \leq \mathbb{P}(C) .
$$


Assim, $\mathbb{P}(C)=0$, no processo sem ativação implicará na morte do processo Firework Discreto. Para o processo sem ativação defina o evento $B_{n}$ : "Partícula $n$ não é atingida pela explosão de nenhuma outra". Então

$$
\mathbb{P}\left(B_{n}\right)=\mathbb{P}\left(\bigcap_{i=1}^{n}\left[R_{n-i}<i\right]\right)=\prod_{i=1}^{n} \mathbb{P}\left(R_{n-i}<i\right)
$$

Como os alcances das explosões são v.a.i.i.d.

$$
\mathbb{P}\left(B_{n}\right)=\prod_{i=1}^{n} \mathbb{P}(R<i) .
$$

Vamos mostrar que se

$$
\sum_{n=1}^{\infty} \mathbb{P}\left(B_{n}\right)=\infty
$$

então $C$ não ocorre com probabilidade 1.

Primeiro, observe que se $i>j$

$$
B_{i} \cap B_{j}=\bigcap_{k=1}^{i-j}\left[R_{i-k}<k\right] \cap \bigcap_{k=1}^{j}\left[R_{j-k}<k\right]
$$

Disto e usando o fato que os alcances das explosões são v.a.i.i.d. segue que

$$
\begin{aligned}
\mathbb{P}\left(B_{i} \cap B_{j}\right) & =\mathbb{P}\left(\bigcap_{k=1}^{i-j}\left[R_{i-k}<k\right] \cap \bigcap_{k=1}^{j}\left[R_{j-k}<k\right]\right)=\prod_{k=1}^{i-j} \mathbb{P}(R<k) \prod_{k=1}^{i} \mathbb{P}(R<k) \\
& =\mathbb{P}\left(B_{i-j}\right) \mathbb{P}\left(B_{j}\right)
\end{aligned}
$$

Porém,

$$
\mathbb{P}\left(B_{i} \cap B_{j}\right)=\mathbb{P}\left(B_{i-j}\right) \mathbb{P}\left(B_{j}\right)
$$

combinado com

$$
\sum_{n=1}^{\infty} \mathbb{P}\left(B_{n}\right)=\infty
$$

implica

$\mathbb{P}\left(B_{n}\right.$ infinitas vezes $)=1$ (Veja Teorema 2 , página 312 em [15]). 
Mas o evento " $B_{n}$ infinitas vezes" garante a não ocorrência do evento $C$. Por fim, resta encontrar condições para 2.2.3. Para isto, o teste de Raabe nos dá a condição

$$
\lim _{n \rightarrow \infty} n \mathbb{P}(R \geq n)<1
$$

ou a condição $L=1$ e existe um $N$ tal que para todo $n \geq N$

$$
\mathbb{P}(R \geq n)<\frac{1}{n-1}
$$

o que conclui a demonstração.

Exemplo 2.2.1.1. Considere o processo Firework Discreto Unidimensional Homogêneo com as variáveis aleatórias $R$ satisfazendo

$$
\mathbb{P}(R=k)=\frac{1}{(k+1)(k+2)} \text { para } k=0,1, \cdots,
$$

Como $\mathbb{P}(R \geq n)=\frac{1}{n+1}$ e $\lim _{n \rightarrow \infty} n \mathbb{P}(R \geq n)=1$ segue do Teorema 2.2 .1 que $\mathbb{P}(V)=0$.

Exemplo 2.2.1.2. Considere o processo Firework Discreto Unidimensional Homogêneo com as variáveis aleatórias $R$ satisfazendo

$$
\mathbb{P}(R=k)=\frac{2}{(k+2)(k+3)} \text { para } k=0,1, \cdots,
$$

Como $\mathbb{P}(R \geq n)=\frac{2}{n+2}$ e $\lim _{n \rightarrow \infty} n \mathbb{P}(R \geq n)=2$ segue do Teorema 2.2 .1 que $\mathbb{P}(V) \geq \frac{1}{3}$.

Corolário 2.2.1.1. Considere o processo Firework Discreto Unidimensional Homogêneo com as variáveis aleatórias $R$ seguindo uma lei de potência, isto é,

$$
\mathbb{P}(R=k)=\frac{Z_{\alpha}}{(k+1)^{\alpha}} \text { para } k=0,1, \cdots,
$$

onde $Z_{\alpha}$ é uma constante de normalização e $\alpha>1$. Neste caso, o ponto crítico é $\alpha=2$. Mais especificamente, o processo sobrevive com probabilidade positiva se

$$
\alpha<2
$$

e morre quase certamente se

$$
\alpha \geq 2
$$


Prova do Corolário 2.2.1.1

Usando o Teste da Integral:

$\frac{1}{(\alpha-1)(n+1)^{\alpha-1}}=\int_{n+2}^{\infty} \frac{1}{x^{\alpha}} d x \leq \sum_{j=n+1}^{\infty} \frac{1}{j^{\alpha}} \leq \int_{n+1}^{\infty} \frac{1}{x^{\alpha}} d x=\frac{1}{(\alpha-1)(n+2)^{\alpha-1}}$

Logo

$$
\frac{Z_{\alpha}}{(\alpha-1)} \frac{1}{(n+1)^{\alpha-1}} \leq \mathbb{P}(R \geq n) \leq \frac{Z_{\alpha}}{(\alpha-1)} \frac{1}{(n+2)^{\alpha-1}}
$$

Donde

$$
\lim _{n \rightarrow \infty} n \mathbb{P}(R \geq n)=\left\{\begin{array}{l}
+\infty \text { se } \alpha<2 \\
\frac{6}{\pi^{2}} \text { se } \alpha=2 \\
0 \text { se } \alpha>2
\end{array}\right.
$$

Daí concluímos a prova aplicando o Teorema 2.2.1.

Corolário 2.2.1.2. Consideremos agora a seguinte variante do Processo Firework Discreto. Cada partícula ao ser ativada tem probabilidade $p$ de emitir uma explosão cujo o alcance tem distribuição de uma variável aleatória R. Assim, com probabilidade 1-p a partícula é ativada mas não emite explosão. Esse processo modificado apresenta a seguinte transição de fase:se

$$
\lim _{n \rightarrow \infty} n \mathbf{P}(R \geq n)>\frac{1}{p},
$$

então existe probabilidade positiva de sobrevivência; se

$$
\lim _{n \rightarrow \infty} n \mathbf{P}(R \geq n)<\frac{1}{p},
$$

então o processo morre quase certamente; se

$$
\lim _{n \rightarrow \infty} n \mathbf{P}(R \geq n)=\frac{1}{p}
$$

e existe um $N$ tal que para todo $n \geq N$

$$
\mathbb{P}(R \geq n)<\frac{p}{n-1},
$$

então o processo morre quase certamente. 
Prova do Corolário 2.2.1.2

Observe que o processo verifica

$\mathbb{P}(R=0)=p \mathbf{P}(R=0)+1-p \mathrm{e}$

$\mathbb{P}(R=k)=p \mathbf{P}(R=k)$, se $k \neq 0$.

Em particular, $\mathbb{P}(\mathrm{R}=0) \in(0,1)$. Então, para concluir basta aplicar o Teorema 2.2.1 para obter o resultado.

Exemplo 2.2.1.3. Considere a variante do processo Firework Discreto Unidimensional Homogêneo dada no Corolário 2.2.1.2 com as variáveis aleatórias $R$ satisfazendo

$$
\mathbb{P}(R=k)=\frac{2}{(k+2)(k+3)} \text { para } k=0,1, \cdots,
$$

e $p=\frac{2}{5}$.

Como $\mathbb{P}(R \geq n)=\frac{2}{n+2}$ e $\lim _{n \rightarrow \infty} n \mathbb{P}(R \geq n)=2<\frac{5}{2}$ segue do Corolário 2.2.1.2 que $\mathbb{P}(V)=0$.

\subsection{O caso Heterogêneo}

No caso heterogêneo, as variáveis $\left\{R_{n}\right\}_{n \in \mathbb{Z}_{+}}$são independentes, mas não são identicamente distribuídas e existem condições para a distância entre partículas consecutivas.

Teorema 2.3.1. Considere o processo Firework Discreto Unidimensional com partículas sobre os inteiros não negativos. Se as marginais do processo satisfazem:

i) $\mathbb{P}\left(R_{i}<1\right) \in(0,1)$, para todo $i$

ii) $\mathbb{P}\left(R_{i} \geq k\right) \leq \mu(R \geq k)$, para alguma $\mu$ tal que $\lim _{n \rightarrow \infty} n \mu(R \geq n)<1$.

Então, o Processo Firework Discreto morre quase-certamente. Em outras palavras, $\mathbb{P}(V)=0$.

Prova do Teorema 2.3.1

Tome $\mathbf{P}$ e $\mu$, medidas de probabilidades tais que

$$
\mathbf{P}\left(R_{i} \geq k\right) \leq \mu(R \geq k), \forall k, \forall i
$$




$$
\lim _{n \rightarrow \infty} n \mu(R \geq n)<1 .
$$

Considere $\left\{R_{i}\right\}_{i \in \mathbb{N}}$ cópias independentes distribuídas segundo $\mathbf{P}$ e $\left\{R_{i}^{*}\right\}_{i \in \mathbb{Z}_{+}}$ cópias independentes distribuídas segundo $\mu$ tais que $R_{i} \leq R_{i}^{*}$ para todo $i$. Agora observe que se o processo morre para uma realização da seqüência $\left\{R_{i}^{*}\right\}_{i \in \mathbb{Z}_{+}}$, também morre para a realização da seqüência $\left\{R_{i}\right\}_{i \in \mathbb{Z}_{+}}$. Disto e do Teorema 2.2.1 segue o resultado.

Exemplo 2.3.1.1. Considere um Processo Firework Discreto sobre os inteiros não-negativos cujas marginais satisfazem

$$
\mathbb{P}\left(R_{n}=k\right)=\frac{e^{-\lambda_{n}} \lambda_{n}^{k}}{k !} \text { para } k=0,1, \cdots,
$$

com $\lambda_{n} \leq \lambda<\infty$ para todo $n$. Então, pelo Teorema 2.3.1 $\mathbb{P}(V)=0$

O resultado a seguir mostra ser possível termos morte quase certa para um processo heterogêneo cujas variáveis aleatórias $R_{n}$ têm esperança infinita para todo $n$.

Teorema 2.3.2. Considere um Processo Firework Discreto Unidimensional cujas marginais satisfazem

i) $\mathbb{P}\left(R_{n}=0\right)=1-a_{n} e$

ii) $\mathbb{P}\left(R_{n}=k\right)=a_{n+k-1}-a_{n+k}$

para alguma sequência não-crescente $\left(a_{n}\right)_{n \in \mathbb{N}}$ onde $a_{0}<1$ e tal que

$\lim _{n \rightarrow \infty} n a_{n}=0$. Então o processo morre quase certamente.

Prova do Teorema 2.3.2

Observe que

$$
V_{n}=V_{n-1} \cap\left(\left[R_{n-1} \geq 1\right] \cup\left[R_{n-2} \geq 2\right] \cup\left[\cdots \cup R_{0} \geq n\right]\right)
$$

Então

$$
\mathbb{P}\left(V_{n}\right) \leq \sum_{k=0}^{n-1} \mathbb{P}\left(R_{k} \geq n-k\right)=\sum_{k=0}^{n-1} a_{n-1}=n a_{n-1} \rightarrow 0 \text { quando } \mathrm{n} \rightarrow \infty .
$$


Exemplo 2.3.2.1. Considere um Processo Firework Discreto sobre os inteiros não-negativos cujas marginais satisfazem

$$
\begin{aligned}
& \mathbb{P}\left(R_{n}=k\right)=\frac{1}{(n+k+1) \ln (n+k+1)}-\frac{1}{(n+k+2) \ln (n+k+2)} \text { para } k \neq 0 \mathrm{e} \\
& \mathbb{P}\left(R_{n}=0\right)=1-\frac{1}{(n+2) \ln (n+2)}
\end{aligned}
$$

Observe que $\mathbb{E}\left(R_{n}\right)=\infty$ para todo $n$, mas $\mathbb{P}(V)=0$

O resultado a seguir exibe uma maneira alternativa de escrever a condição para sobrevivência com probabilidade positiva do processo Firework Discreto heterogêneo.

Teorema 2.3.3. Suponha um processo Firework Discreto não homogêneo com partículas nas posições $0=u_{0}<u_{1}<u_{2}<\ldots$ tais que $u_{n+1}-u_{n} \leq m$. Se $\mathbb{P}\left(R_{n}<m\right) \in(0,1)$ para todo $n$ e existe $t \geq 1$ tal que

$$
\sum_{n=0}^{\infty}\left[\mathbb{P}\left(R_{n}<t m\right)\right]^{t}<\infty
$$

então o processo sobrevive com probabilidade positiva.

Prova do Teorema 2.3.3

Observe que para $n>t$ temos

$$
\begin{aligned}
a_{n} & :=\prod_{j=0}^{n} \mathbb{P}\left(R_{n-j}<(j+1) m\right) \leq \prod_{j=0}^{t-1} \mathbb{P}\left(R_{n-j}<t m\right) \\
& \leq\left[\max _{j \in\{0, \ldots, t-1\}}\left[\mathbb{P}\left(R_{n-j}<t m\right)\right]\right]^{t} .
\end{aligned}
$$

Por outro lado

$$
\sum_{n=t}^{\infty}\left[\mathbb{P}\left(R_{n}<t m\right)\right]^{t}<\infty \Rightarrow \sum_{n=t}^{\infty}\left[\max _{j \in\{0, \ldots, t-1\}}\left[\mathbb{P}\left(R_{n-j}<t m\right)\right]\right]^{t}<\infty .
$$

Assim, a série com termos $a_{n}$ é convergente e podemos aplicar a Proposição 2.1.0.2 para obter o resultado.

Exemplo 2.3.3.1. Considere um Processo Firework Discreto sobre os inteiros não-negativos cujas marginais satisfazem

$$
\mathbb{P}\left(R_{n}=k\right)=\frac{k}{n+2} \text { para } k=0,1, \cdots, n+1
$$


Temos $\sum_{n=t}^{\infty}\left(\frac{t}{n+1}\right)^{t}<\infty$ para $t \geq 2$.Segue $\mathbb{P}(V)>0$ pelo Teorema 2.3.3.

Exemplo 2.3.3.2. Considere um Processo Firework Discreto sobre os inteiros não-negativos cujas marginais satisfazem

$$
\mathbb{P}\left(R_{n}=0\right)=\frac{1}{(n+2)^{2}} \text { e } \mathbb{P}\left(R_{n}=1\right)=1-\frac{1}{(n+2)^{2}}
$$

Observe que $\mathbb{E}\left(R_{n}\right)<1$ para todo $n$, mas $\mathbb{P}(V)=\frac{1}{2}$

Teorema 2.3.4. Suponha um Processo Firework Discreto Unidimensional não homogêneo com partículas nas posições $0=u_{0}<u_{1}<u_{2}<\ldots$, tais que $u_{n+1}-u_{n} \leq m$. Se as marginais do processo são tais que $\mathbb{P}\left(R_{n}<m\right) \in(0,1)$ para todo $n$ e

$$
\mathcal{P}(R \geq k)-\mathbb{P}\left(R_{n} \geq k\right) \leq b_{k}
$$

para todo $k \geq 0$ e todo $n \geq 0$, onde

$$
\lim _{n \rightarrow \infty} n\left[\mathcal{P}(R \geq n)-b_{n}\right]>m \text { com } \lim _{n \rightarrow \infty} b_{n}=0
$$

o processo sobrevive com probabilidade positiva.

Prova do Teorema 2.3.4

Observe que

$$
\begin{gathered}
a_{n}:=\prod_{j=0}^{n} \mathbb{P}\left(R_{n-j}<(j+1) m\right) \leq \prod_{j=0}^{n}\left[\mathcal{P}(R<(j+1) m)+b_{(j+1) m}\right] . \\
a_{n} \leq \prod_{j=0}^{n}\left[\mathcal{P}(R<(j+1) m)+b_{(j+1) m}\right] .:=r_{n}
\end{gathered}
$$

Mas

$$
n\left(\frac{r_{n}}{r_{n+1}}-1\right)=\frac{n\left[\mathcal{P}(R \geq(n+2) m)-b_{(n+2) m}\right]}{\mathcal{P}(R<(n+2) m)+b_{(n+2) m}} .
$$

Logo, das hipóteses segue que

$$
\lim _{n \rightarrow \infty} n\left(\frac{r_{n}}{r_{n+1}}-1\right)>1 .
$$

Assim, a série com termos $\mathrm{a}_{n}$ é convergente e podemos aplicar a Proposição 2.1.0.2 para obter o resultado. 


\subsection{Processo Firework Discreto Unidimensional Re- verso}

Considere a seguinte variante do processo Firework Discreto, que chamaremos Firework Reverso. Consideramos um conjunto de partículas sobre os inteiros não negativos. No instante 0 , todas as partículas estão inativas, exceto aquela na origem que é inicialmente ativada. Uma partícula ao ser ativada assim permanece para sempre. A cada partícula é associada uma variável aleatória que representa o seu alcance de ativação. Assim, se para uma dada partícula $n$ o alcance de ativação é $R_{n}$, ela se tornará ativa se alguma partícula a sua esquerda, cuja distância a ela é inferior ou igual a $R_{n}$ se tornar ativada. Os alcances de ativação $\left\{R_{n}\right\}_{n \in \mathbb{Z}_{+}^{*}}$ são variáveis aleatórias independentes. Assim como no Processo Firework Discreto apresentamos as versões homogênea e heterogênea e estudamos as condições de sobrevivência. Dizemos que o processo sobrevive se infinitas partículas forem ativadas ao longo do processo. No caso homogêneo apresentamos transição de fase.

Consideremos as seguintes definições:

Definição 2.4.0.1. Sejam $\left\{R_{n}\right\}_{n \in \mathbb{Z}_{+}^{*}}$ variáveis aleatórias independentes assumindo valores nos reais não negativos, com distribuição $\left\{\mathbf{P}_{\mathbf{n}}\right\}_{n \in \mathbb{Z}_{+}^{*}}$ respectivamente. Defina

$$
\mathbb{P}=\prod_{n=1}^{\infty} \mathbf{P}_{n}
$$

$\mathbb{P}$ é a medida de probabilidade para o Processo Firework Reverso.

Observação. $\mathbb{P}\left(R_{n}=k\right)=\mathbf{P}_{n}\left(R_{n}=k\right)$

Definição 2.4.0.2. Considere o evento $V$ : "O processo sobrevive ". Observe que

$$
V=\bigcap_{n=0}^{\infty} \bigcup_{j=1}^{\infty}\left[R_{n+j} \geq j\right]
$$

Apresentaremos alguns resultados úteis na prova dos resultados.

Lema 2.4.0.1.

$$
\mathbb{P}\left(\bigcap_{n=0}^{\infty} \bigcup_{j=1}^{\infty}\left[R_{n+j} \geq j\right]\right) \geq \prod_{n=0}^{\infty} \mathbb{P}\left(\bigcup_{j=1}^{\infty}\left[R_{n+j} \geq j\right]\right)
$$


Prova do Lema 2.4.0.1

Observe que os eventos

$$
\left\{\bigcup_{j=1}^{\infty}\left[R_{n+j} \geq j\right]\right\}_{n \in \mathbb{Z}_{+}}
$$

são eventos crescentes. Usando a desigualdade FKG (Lema 5.1.0.1) e o fato de que a intersecção de eventos crescentes é um evento crescente temos:

$$
\mathbb{P}\left(\bigcap_{n=0}^{n_{0}} \bigcup_{j=1}^{\infty}\left[R_{n+j} \geq j\right]\right) \geq \prod_{n=0}^{n_{0}} \mathbb{P}\left(\bigcup_{j=1}^{\infty}\left[R_{n+j} \geq j\right]\right)
$$

para todo $n_{0}$ inteiro positivo. Tomando o limite $n_{0} \rightarrow \infty$ e usando a Continuidade da Probabilidade temos o resultado.

Lema 2.4.0.2.

$$
\mathbb{P}(V) \geq \prod_{n=0}^{\infty}\left[1-\prod_{j=1}^{\infty}\left[1-\mathbb{P}\left(R_{n+j} \geq j\right)\right]\right]
$$

Prova do Lema 2.4.0.2

Temos

$$
\begin{gathered}
V=\bigcap_{n=0}^{\infty} \bigcup_{j=1}^{\infty}\left[R_{n+j} \geq j\right] \\
\mathbb{P}(V)=\mathbb{P}\left(\bigcap_{n=0}^{\infty} \bigcup_{j=1}^{\infty}\left[R_{n+j} \geq j\right]\right) \geq \prod_{n=0}^{\infty} \mathbb{P}\left(\bigcup_{j=1}^{\infty}\left[R_{n+j} \geq j\right]\right) \\
=\prod_{n=0}^{\infty}\left[1-\mathbb{P}\left(\bigcap_{j=1}^{\infty}\left[R_{n+j}<j\right]\right)\right]
\end{gathered}
$$

Onde a desigualdade é obtida pelo Lema 2.4.0.1.O resultado segue da independência das variáveis $R_{i}$.

\subsubsection{O caso Reverso Homogêneo}

No caso homogêneo os alcances de ativação são variáveis aleatórias independentes e identicamente distribuídas. 


\subsection{PROCESSO FIREWORK DISCRETO UNIDIMENSIONAL REVERSO21}

Teorema 2.4.1. Considere o Processo Firework Reverso Homogêneo e suponha $\mathbb{P}(R=0) \in(0,1)$. Se $\mathbb{E}(R)=\infty$, então $\mathbb{P}(V)=1$. Por outro lado, se $\mathbb{E}(R)<\infty$, então $\mathbb{P}(V)=0$.

Prova do Teorema 2.4.1

Do Lema 2.4.0.2,

$$
\mathbb{P}(V) \geq \prod_{n=0}^{\infty}\left[1-\prod_{j=1}^{\infty}[1-\mathbb{P}(R \geq j)]\right]
$$

Agora, observe que

$\mathbb{E}(R)=\sum_{j=1}^{\infty} \mathbb{P}(R \geq j)=\infty \Rightarrow \prod_{j=1}^{\infty}[1-\mathbb{P}(R \geq j)]=0 \Rightarrow \mathbb{P}(V) \geq 1 \Rightarrow \mathbb{P}(V)=1$.

Para a outra parte, suponha $\mathbb{E}(R)<\infty$. Considere então a seguinte variante do Processo Firework Discreto Homogêneo. Colocamos exatamente uma partícula sobre cada inteiro. Inicialmente todas as partículas sobre os inteiros não positivos estão ativadas. Os alcances de explosão são as variáveis aleatórias $\left\{\mathcal{R}_{n}\right\}_{n \in \mathbb{Z}}$. Suponha que toda distribuição $\mathcal{R}_{n}$ dessa família tenha a mesma lei de uma variável aleatória $R_{n}$ do Processo Reverso Homogêneo. Assim, $\mathbb{P}\left(R_{n}=j\right)=\mathbb{P}\left(\mathcal{R}_{m}=j\right)$ para todos $j, n$ inteiros não negativos e $m$ inteiro. Aqui cometemos um abuso de notação já que as variáveis $\left\{R_{n}\right\}_{n \in \mathbb{Z}_{+}}$ e $\left\{\mathcal{R}_{n}\right\}_{n \in \mathbb{Z}_{+}}$não estão no mesmo espaço de probabilidade.

Para este Processo Firework Discreto Homogêneo descrito sejam os eventos: $\mathcal{V}_{n}$ "Partícula em n é atingida"e $\mathcal{V}$, o evento "Processo sobrevive". Neste modelo, sobreviver significa acordar infinitas partículas sobre os inteiros positivos. Observe que

$$
\mathcal{V}=\bigcap_{n=0}^{\infty} \bigcup_{j=0}^{\infty}\left[\mathcal{R}_{n-j} \geq j+1\right]
$$

Proposição 2.4.1.1. Se $\mathbb{P}(\mathcal{R}=0) \in(0,1)$ e $\mathbb{E}(\mathcal{R})<\infty$, então $\mathbb{P}(\mathcal{V})=0$

Prova da Proposição 2.4.1.1

Defina os seguintes eventos

$$
\mathcal{A}_{n}:=\bigcup_{i=-\infty}^{n-1}\left\{\mathcal{R}_{i} \geq 2 n-i\right\}
$$


e

$$
\mathcal{B}_{n}:=\bigcup_{i=n}^{2 n-1}\left\{\mathcal{R}_{i} \geq 2 n-i\right\}
$$

Observe que

$$
\begin{gathered}
\mathcal{V}_{2 n} \subseteq \mathcal{V}_{n} \cap\left[\mathcal{A}_{n} \cup \mathcal{B}_{n}\right] \\
\mathbb{P}\left(\mathcal{V}_{2 n}\right) \leq \mathbb{P}\left(\mathcal{V}_{n} \cap\left[\mathcal{A}_{n} \cup \mathcal{B}_{n}\right]\right)=\mathbb{P}\left(\left[\mathcal{V}_{n} \cap \mathcal{A}_{n}\right] \cup\left[\mathcal{V}_{n} \cap \mathcal{B}_{n}\right]\right) \leq \mathbb{P}\left(\mathcal{A}_{n} \cup\left[\mathcal{V}_{n} \cap \mathcal{B}_{n}\right]\right) \\
\mathbb{P}\left(\mathcal{V}_{2 n}\right) \leq \mathbb{P}\left(\mathcal{A}_{n}\right)+\mathbb{P}\left(\mathcal{B}_{n} \cap \mathcal{V}_{n}\right)=\mathbb{P}\left(\mathcal{A}_{n}\right)+\mathbb{P}\left(\mathcal{B}_{n}\right) \mathbb{P}\left(\mathcal{V}_{n}\right)
\end{gathered}
$$

$\mathrm{Na}$ última igualdade usamos o fato dos eventos $B_{n}$ e $V_{n}$ serem independentes.

Lema 2.4.1.1. Nas hipóteses da Proposição 2.4.1.1

$$
\lim _{n \rightarrow \infty} \mathbb{P}\left(\mathcal{A}_{n}\right)=0
$$

Prova do Lema 2.4.1.1

$$
\begin{aligned}
\mathbb{P}\left(\mathcal{A}_{n}\right) & =\mathbb{P}\left(\bigcup_{i=-\infty}^{n-1}\left\{\mathcal{R}_{2 n-i} \geq i\right\}\right) \leq \sum_{i=-\infty}^{n-1} \mathbb{P}\left(\mathcal{R}_{i} \geq 2 n-i\right) \\
& =\sum_{i=n+1}^{\infty} \mathbb{P}\left(\mathcal{R}_{2 n-i} \geq i\right)=\sum_{i=n+1}^{\infty} \mathbb{P}(\mathcal{R} \geq i) \rightarrow 0 \text {, quando } n \rightarrow \infty
\end{aligned}
$$

Lema 2.4.1.2. Nas hipóteses da Proposição 2.4.1.1 existe $\lambda \in(0,1)$ tal que para todo $n$

$$
\mathbb{P}\left(\mathcal{B}_{n}\right) \leq \lambda
$$

Prova do Lema 2.4.1.2

$$
\begin{aligned}
\mathbb{P}\left(\mathcal{B}_{n}\right) & =\mathbb{P}\left(\bigcup_{i=n}^{2 n-1}\left\{\mathcal{R}_{i} \geq 2 n-i\right\}\right)=1-\mathbb{P}\left(\bigcap_{i=n}^{2 n-1}\left\{\mathcal{R}_{i}<2 n-i\right\}\right) \\
& =1-\prod_{i=n}^{2 n-1} \mathbb{P}\left(\mathcal{R}_{i}<2 n-i\right)=1-\prod_{i=1}^{n} \mathbb{P}(\mathcal{R}<i) \leq 1-\prod_{i=1}^{\infty} \mathbb{P}(\mathcal{R}<i) .
\end{aligned}
$$

Assim, o Lema 5.1.0.2 (ver apêndice) e $\mathbb{E}(\mathcal{R})<\infty$ nos garante que existe $\lambda \in(0,1)$ tal que

$$
\mathbb{P}\left(\mathcal{B}_{n}\right) \leq \lambda
$$

para todo n, concluindo a demonstração. 


\subsection{PROCESSO FIREWORK DISCRETO UNIDIMENSIONAL REVERSO23}

Lema 2.4.1.3. Nas hipóteses da Proposição 2.4.1.1

$$
\mathbb{P}\left(\mathcal{A}_{n}\right) \geq \mathbb{P}\left(\mathcal{A}_{n+1}\right) \text { para todo } n .
$$

Prova do Lema 2.4.1.3

$$
\begin{aligned}
\mathbb{P}\left(\mathcal{A}_{n}\right) & =\mathbb{P}\left(\bigcup_{i=-\infty}^{n-1}\left\{\mathcal{R}_{2 n-i} \geq i\right\}\right)=1-\mathbb{P}\left(\bigcap_{i=-\infty}^{n-1}\left\{\mathcal{R}_{2 n-i}<i\right\}\right) \\
& =1-\prod_{j=n+1}^{\infty} \mathbb{P}(\mathcal{R}<i) .
\end{aligned}
$$

Analogamente, $\mathbb{P}\left(\mathcal{A}_{n+1}\right)=1-\prod_{j=n+2}^{\infty} \mathbb{P}(\mathcal{R}<i)$.

Logo,

$\mathbb{P}\left(\mathcal{A}_{n}\right)=1-\prod_{j=n+1}^{\infty} \mathbb{P}(\mathcal{R}<i) \geq 1-\prod_{j=n+2}^{\infty} \mathbb{P}(\mathcal{R}<i)=\mathbb{P}\left(\mathcal{A}_{n+1}\right)$.

Voltemos a prova da Proposição 2.4.1.1. Observe que usando o Lema 2.4.1.2 temos

$$
\mathbb{P}\left(\mathcal{V}_{2 n}\right) \leq \mathbb{P}\left(\mathcal{A}_{n}\right)+\lambda \mathbb{P}\left(\mathcal{V}_{n}\right) \text { para todo } n
$$

Usando o Lema 2.4.1.3, obtemos

$$
\begin{aligned}
& \mathbb{P}\left(\mathcal{V}_{2 n}\right) \leq \mathbb{P}\left(\mathcal{A}_{n}\right)+\lambda \mathbb{P}\left(\mathcal{V}_{n}\right) \\
& \mathbb{P}\left(\mathcal{V}_{4 n}\right) \leq \mathbb{P}\left(\mathcal{A}_{2 n}\right)+\lambda \mathbb{P}\left(\mathcal{V}_{2 n}\right) \leq \mathbb{P}\left(\mathcal{A}_{n}\right)+\lambda \mathbb{P}\left(\mathcal{V}_{2 n}\right) \\
& \mathbb{P}\left(\mathcal{V}_{8 n}\right) \leq \mathbb{P}\left(\mathcal{A}_{4 n}\right)+\lambda \mathbb{P}\left(\mathcal{V}_{4 n}\right) \leq \mathbb{P}\left(\mathcal{A}_{n}\right)+\lambda \mathbb{P}\left(\mathcal{V}_{4 n}\right) \\
& \vdots \\
& \mathbb{P}\left(\mathcal{V}_{2^{m} n}\right) \leq \mathbb{P}\left(\mathcal{A}_{2^{m-1} n}\right)+\lambda \mathbb{P}\left(\mathcal{V}_{2^{m-1} n}\right) \leq \mathbb{P}\left(\mathcal{A}_{n}\right)+\lambda \mathbb{P}\left(\mathcal{V}_{2^{m-1} n}\right)
\end{aligned}
$$

Logo,

$$
\begin{aligned}
& \mathbb{P}\left(\mathcal{V}_{2^{m} n}\right) \leq \mathbb{P}\left(\mathcal{A}_{n}\right)+\lambda \mathbb{P}\left(\mathcal{A}_{n}\right)+\lambda^{2} \mathbb{P}\left(\mathcal{A}_{n}\right)+\cdots \lambda^{m-1} \mathbb{P}\left(\mathcal{A}_{n}\right)+\lambda^{m} \mathbb{P}\left(\mathcal{V}_{n}\right) \\
& \mathbb{P}\left(\mathcal{V}_{2^{m}}\right) \leq\left(1+\lambda+\lambda^{2}+\cdots+\lambda^{m-1}\right) \mathbb{P}\left(\mathcal{A}_{n}\right)+\lambda^{m} \\
& \mathbb{P}\left(\mathcal{V}_{2^{m} n}\right) \leq \frac{\left(1-\lambda^{m}\right)}{(1-\lambda)} \mathbb{P}\left(\mathcal{A}_{n}\right)+\lambda^{m}
\end{aligned}
$$


Tome $n=2^{m}$. Temos

$$
\mathbb{P}\left(\mathcal{V}_{4^{m}}\right) \leq \frac{\left(1-\lambda^{m}\right)}{(1-\lambda)} \mathbb{P}\left(\mathcal{A}_{2^{m}}\right)+\lambda^{m} .
$$

Tomando $m \rightarrow \infty$ segue do Lema 2.4.1.1 que

$$
\lim _{m \rightarrow \infty} \mathbb{P}\left(\mathcal{V}_{4^{m}}\right)=0
$$

Agora observe que para todo $n \geq 1$ existe $m(n) \geq 1$ tal que $\mathcal{V}_{4^{m+1}} \subset \mathcal{V}_{n} \subset$ $\mathcal{V}_{4^{m}}$ e $\lim _{n \rightarrow \infty} m(n)=\infty$. Isso combinado com 2.4.2 nos dá pela Continuidade da Probabilidade que $\mathbb{P}(\mathcal{V})=0$.

Agora vamos finalizar a prova do Teorema 2.4.1. Observe que

$$
\mathbb{P}\left(\bigcap_{n=0}^{\infty} \bigcup_{j=0}^{\infty}\left[\mathcal{R}_{n-j} \geq j+1\right]\right) \geq \mathbb{P}\left(\bigcap_{n=0}^{\infty} \bigcup_{j=0}^{\infty}\left[R_{n+j+1} \geq j+1\right]\right)
$$

Para verificar este fato observe o seguinte. Olhando para o evento da esquerda, para todo $n$ fixado, é preciso que algum raio à esquerda (desde $n$ até $-\infty$ ) alcance a distância da partícula até $n+1$. Do mesmo modo, olhando para o evento da direita, para todo $n$ fixado, é preciso que algum raio à direita (desde $n+1$ até $\infty$ ) alcance a distância da partícula até $n$.

Mas,

$$
V=\bigcap_{n=0}^{\infty} \bigcup_{j=0}^{\infty}\left[R_{n+j+1} \geq j+1\right]
$$

Logo, $\mathbb{P}(V)=0$.

Aqui uma questão interessante é respondida. Será que os processos Firework Discreto Homogêneo e Firework Reverso Homogêneo apresentam resultados distintos quanto a sobrevivência? Pelos resultados apresentados, a resposta é sim. Por exemplo, consideremos estes dois processos com alcances satisfazendo

$$
\mathbb{P}(R=k)=\frac{6}{[\pi(k+1)]^{2}} .
$$

Nesse caso, o processo Firework Discreto Homogêneo morre quase certamente. Por outro lado, o Processo Firework Reverso Homogêneo sobrevive com probabilidade 1. 


\subsection{PROCESSO FIREWORK DISCRETO UNIDIMENSIONAL REVERSO25}

\subsubsection{O caso Reverso Heterogêneo}

No caso heterogêneo os alcances de ativação são variáveis aleatórias independentes não identicamente distribuídas satisfazendo determinadas condições.

Teorema 2.4.2. Considere o Processo Firework Reverso Heterogêneo. Suponha $\mathbb{P}\left(R_{n}=0\right) \in(0,1)$ para todo $n$. Temos:

$$
\begin{aligned}
& \text { i) } \mathbb{P}(V)=1 \text { se e somente se } \sum_{k=1}^{\infty} \mathbb{P}\left(R_{n+k} \geq k\right)=\infty \text { para todo } n . \\
& \text { ii) } \mathbb{P}(V)>0 \text { se } \sum_{n=1}^{\infty} \prod_{k=1}^{\infty} \mathbb{P}\left(R_{n+k}<k\right)<\infty \\
& \text { iii) } \mathbb{P}(V)=0 \text { se existe uma medida de probabilidade } \mathbf{P}, \text { tal que } \\
& \mathbb{P}\left(R_{n} \geq k\right) \leq \mathbf{P}(R \geq k), \forall k \text { e } \forall n \text { e } \mathbb{E}_{\mathbf{P}}(R)<\infty .
\end{aligned}
$$

Prova do Teorema 2.4.2

Do Lema 2.4.0.2,

$$
\mathbb{P}(V) \geq \prod_{n=1}^{\infty}\left[1-\prod_{k=1}^{\infty}\left[1-\mathbb{P}\left(R_{n+k} \geq k\right)\right]\right]
$$

Para que o produto à direita seja 1 é preciso que

$$
\prod_{k=1}^{\infty}\left[1-\mathbb{P}\left(R_{n+k} \geq k\right)\right]=0
$$

para todo $n$. Pelo Lema 5.1.0.2 isto irá ocorrer se e somente se

$$
\sum_{k=1}^{\infty} \mathbb{P}\left(R_{n+k} \geq k\right)=\infty \text { para todo } n .
$$

Por outro lado,

$$
\mathbb{P}(V) \leq 1-\prod_{k=1}^{\infty} \mathbb{P}\left(R_{n+k}<k\right) \text { para todo } n .
$$

Desses dois fatos estabelecemos $i$ ).

Para provar $i$ ) observe que o Lema 5.1.0.2 nos garante que para o processo ter chance positiva de sobrevivência basta

$$
\sum_{n=1}^{\infty} \prod_{k=1}^{\infty} \mathbb{P}\left(R_{n+k}<k\right)<\infty .
$$


Para provar $i$ ii) sejam $\mathbb{P}$ e $\mathbf{P}$, medidas de probabilidades tais que

$$
\mathbb{P}\left(R_{n} \geq k\right) \leq \mathbf{P}(R \geq k), \forall k \text { e } \forall n,
$$

e

$$
\mathbb{E}_{\mathbf{P}}(R)<\infty .
$$

Considere $\left\{R_{n}\right\}_{n \in \mathbb{Z}_{+}^{*}}$ cópias independentes distribuídas segundo $\mathbb{P}$ e $\left\{R_{n}^{*}\right\}_{n \in \mathbb{Z}_{+}^{*}}$ cópias independentes distribuídas segundo $\mathbf{P}$ tais que $R_{n} \leq R_{n}^{*}$ para todo $n>0$. Agora observe que se o processo morre para uma realização da seqüência $\left\{R_{n}^{*}\right\}_{n \in \mathbb{Z}_{+}^{*}}$, também morre para a realização da seqüência $\left\{R_{n}\right\}_{n \in \mathbb{Z}_{+}^{*}}$. Isto e o Teorema 2.4 .1 nos dá o resultado desejado.

Exemplo 2.4.2.1. Considere um Processo Firework Reverso sobre os inteiros não-negativos com $R_{n} \sim$ Geométrica $\left(1-\frac{1}{n+1}\right)$, para todo $n>0$. Pelo Teorema 2.4.2 $\mathbb{P}(V)=1$.

Exemplo 2.4.2.2. Considere um Processo Firework Reverso sobre os inteiros não-negativos cujas marginais satisfazem

$$
\mathbb{P}\left(R_{n}=0\right)=\frac{1}{(n+1)^{2}} \text { e } \mathbb{P}\left(R_{n}=1\right)=1-\frac{1}{(n+1)^{2}}
$$

Pelo Teorema 2.4.2 $\mathbb{P}(V)>0$.

Exemplo 2.4.2.3. Considere um Processo Firework Reverso sobre os inteiros não-negativos com $R_{n} \sim \operatorname{Poisson}\left(\lambda_{n}\right), \lambda_{n} \leq \lambda<\infty$ para todo $n$. Pelo Teorema 2.4.2 $\mathbb{P}(V)=0$. 


\section{Capítulo 3}

\section{Processo Firework Contínuo Unidimensional}

A dinâmica do processo Firework Contínuo é a seguinte. Consideramos partículas distribuídas sobre a semirreta dos reais positivos nas posições $u_{n}$, onde $n$ indica que a partícula é a n-ésima mais próxima da origem. Nos referimos a ela como partícula $n$. A partícula que dá início ao processo se encontra na posição $u_{0}=0$, isto é, sobre a origem. No instante $t=0$, todas as partículas estão inativas, exceto aquela na origem que é inicialmente ativada e dá início ao processo. Uma partícula $n$ ao ser ativada, assim permanece durante um tempo aleatório $T_{n}$. Durante este tempo a partícula $n$ emite explosões independentes com alcances aleatórios e finitos $\left\{\bar{R}_{n}^{j}\right\}_{j \in \mathbb{Z}_{+}^{*}}$ As explosões ocorrem em instantes pontuais aleatórios de modo que o tempo entre explosões consecutivas é exponencial com parâmetro $\mu_{n}$. Quando uma partícula inativa é atingida por uma explosão ela se torna ativa. Cada partícula somente pode ser ativada uma única vez, isto é, se uma partícula ativa tem seu tempo de ativação encerrado, ela não pode ser ativada novamente, mesmo que atingida pela explosão de outra partícula.

Consideramos dois casos: o homogêneo e o heterogêneo. No caso homogêneo os alcances de explosões são v.a.i.i.d.. Também são v.a.i.i.d. os tempos de ativação e os tempos entre explosões. No caso heterogêneo todos estas variáveis são independentes, entretanto pelo menos um dos conjuntos de variáveis é não identicamente distribuído ou as partículas não estão necessariamente sobre $\mathbb{Z}_{+}$.

Definição 3.0.2.1. Seja $N$ uma variável aleatória discreta assumindo valores nos inteiros não negativos. Defina

$$
\varphi_{N}(s):=\mathbb{E}\left(s^{N}\right) \text {, para } s \in(-1 ; 1) .
$$


$\varphi_{N}$ é a função geradora de probabilidade da variável aleatória $N$.

Definição 3.0.2.2. Seja $T$ uma variável aleatória qualquer. Defina

$$
M_{T}(s):=\mathbb{E}\left(e^{s T}\right) \text {, para } s \text { tal que } \mathbb{E}\left(e^{s T}\right)<\infty .
$$

$M_{T}$ é a função geradora de momentos da variável aleatória $T$.

\subsection{Resultados preliminares}

Inicialmente consideramos uma versão mais simples do processo Firework a qual chamaremos Firework com número aleatório de tentativas de ativação. Nesta versão, uma partícula $n$ ao ser ativada emite explosões uma quantidade aleatória de vezes. Essa quantidade é dada por uma variável aleatória $N_{n}$. A i-ésima tentativa de ativar vizinhos realizada pela partícula em j é dada pela variável aleatória $\bar{R}_{j}^{i}$. As tentativas de ativação são sempre v.a.i.i.d..

Proposição 3.1.0.1. Suponha um processo Firework com número aleatório de tentativas de ativação em sua versão homogênea. Defina

$$
f_{\bar{R}, N}(n):=n\left\{1-\varphi_{N}(\mathbb{P}(\bar{R}<n))\right\}
$$

Então

Se $\lim _{n \rightarrow \infty} f_{\bar{R}, N}(n)<1$ então o processo morre quase certamente.

Se $\lim _{n \rightarrow \infty} f_{\bar{R}, N}(n)=1$ e existe $N_{0}$ tal que para todo $n \geq N_{0}$,

$$
\varphi_{N}(\mathbb{P}(\bar{R}<n))>\frac{n-2}{n-1},
$$

então o processo morre quase certamente.

Se $\lim _{n \rightarrow \infty} f_{\bar{R}, N}(n)>1$ então o processo tem probabilidade positiva de sobreviver $e$

$$
\mathbb{P}(V) \geq \prod_{i=0}^{\infty}\left[1-\varphi_{N}\left(\prod_{j=0}^{i} \mathbb{P}(\bar{R}<j+1)\right)\right]
$$


Prova da Proposição 3.1.0.1

Primeiro, observe que a evolução do processo não é alterada se admitirmos que todas as explosões realizadas por uma partícula são instantâneas ao momento de ativação. Associamos cada realização desse Processo a uma realização do Processo Firework Discreto Homogêneo de modo que

$$
(R \geq k)=\left(\max \left\{\bar{R}^{1}, \cdots, \overline{R^{N}}\right\} \geq k\right)
$$

Agora observe que

$$
\left(\max \left\{\bar{R}^{1}, \cdots, \overline{R^{N}}\right\} \geq k\right)=\bigcup_{i=1}^{\infty}\left(\max \left\{\overline{R^{1}}, \cdots, \overline{R^{N}}\right\} \geq k, N=i\right)
$$

Mas

$$
\mathbb{P}\left(\max \left\{\bar{R}^{1}, \cdots, \overline{R^{N}}\right\} \geq k, N=i\right)=\mathbb{P}(N=i) \mathbb{P}\left(\bar{R}^{1} \geq k \cup \cdots \cup \bar{R}^{i} \geq k\right)
$$

Por outro lado

$$
\begin{aligned}
& \mathbb{P}\left(\bar{R}^{1} \geq k \cup \cdots \cup \bar{R}^{i} \geq k\right)=1-\mathbb{P}\left(\bar{R}^{1}<k \cap \cdots \cap \bar{R}^{i}<k\right)= \\
& 1-\prod_{j=1}^{i} \mathbb{P}\left(\bar{R}^{j}<k\right)=1-[\mathbb{P}(\bar{R}<k)]^{i} .
\end{aligned}
$$

Daí

$$
\mathbb{P}\left(\max \left\{\bar{R}^{1}, \cdots, \overline{R^{N}}\right\} \geq k, N=i\right)=\mathbb{P}(N=i)\left[1-[\mathbb{P}(\bar{R}<k)]^{i}\right]
$$

Logo

$$
\begin{aligned}
\mathbb{P}(R \geq k) & =\sum_{i=0}^{\infty} \mathbb{P}\left(\max \left\{\bar{R}^{1}, \cdots, \overline{R^{N}}\right\} \geq k, N=i\right) \\
& =\sum_{i=1}^{\infty} \mathbb{P}(N=i)\left[1-[\mathbb{P}(\bar{R}<k)]^{i}\right] \\
& \left.=\sum_{i=1}^{\infty} \mathbb{P}(N=i)-\sum_{i=1}^{\infty} \mathbb{P}(N=i)[\mathbb{P}(\bar{R}<k)]^{i}\right] \\
& =1-\mathbb{P}(N=0)-\left\{\mathbb{E}\left[(\mathbb{P}(\bar{R}<k))^{N}\right]-\mathbb{P}(N=0)\right\} \\
& =1-\mathbb{E}\left[(\mathbb{P}(\bar{R}<k))^{N}\right]
\end{aligned}
$$


Logo, considerando um processo Firework Discreto homogêneo com distribuição de probabilidade satisfazendo

$$
\mathbb{P}(R \geq n)=1-\mathbb{E}\left[(\mathbb{P}(\bar{R}<n))^{N}\right]=1-\varphi_{N}(\mathbb{P}(\bar{R}<n))
$$

nós obtemos o resultado desejado.

Proposição 3.1.0.2. Suponha um processo Firework com um número aleatório de tentativas de ativação em sua versão não homogênea.

Se existe $m \in \mathbb{Z}_{+}$tal que as posições das partículas satisfazem $u_{n+1}-u_{n} \leq m$ $e$

$$
\sum_{n=0}^{\infty} \prod_{j=0}^{n} \varphi_{N-j}\left(\mathbb{P}\left(\bar{R}_{n-j}<(j+1) m\right)\right)<\infty,
$$

então o processo sobrevive com probabilidade positiva. Nesse caso,

$$
\mathbb{P}(V) \geq \prod_{n=0}^{\infty}\left[1-\prod_{j=0}^{n} \varphi_{N-j}\left(\mathbb{P}\left(\bar{R}_{n-j}<(j+1) m\right)\right)\right] .
$$

Por outro lado, se existe $k \in \mathbb{Z}_{+}$tal que as posições das partículas satisfazem $u_{n+1}-u_{n} \geq k$ e as marginais do processo satisfazem:

1.

$$
\varphi_{N_{i}}\left(\mathbb{P}\left(\bar{R}_{i}<k\right)\right) \in(0,1)
$$

2.

$$
\lim _{n \rightarrow \infty} \prod_{i=0}^{n-1} \varphi_{N_{i}}\left(\mathbb{P}\left(\bar{R}_{i}<k(2 n-i)\right)\right)=1
$$

3.

$$
\lim _{n \rightarrow \infty} \prod_{i=n}^{2 n-1} \varphi_{N_{i}}\left(\mathbb{P}\left(\bar{R}_{i}<k(2 n-i)\right)\right)>0 ;
$$

então o processo morre quase certamente.

Prova da Proposição 3.1.0.2

Analogamente ao último resultado, associamos cada realização do Processo a uma realização do Processo Firework Discreto Heterogêneo de modo que

$$
\left(R_{i} \geq k\right)=\left(\max \left\{\bar{R}_{i}^{1}, \cdots, \overline{R_{i}^{N}}\right\} \geq k\right) .
$$

O que nos dá

$$
\mathbb{P}\left(R_{i} \geq n\right)=1-\mathbb{E}\left[\left(\mathbb{P}\left(\bar{R}_{i}<n\right)\right)^{N_{i}}\right]=1-\varphi_{N_{i}}\left(\mathbb{P}\left(\bar{R}_{i}<n\right)\right) .
$$

O resultado segue pelas Proposições 2.1.0.2 e 2.1.0.1. 


\subsection{O caso Homogêneo}

No caso homogêneo os alcances de explosões são v.a.i.i.d.. Também são v.a.i.i.d. os tempos de ativação e os tempos entre explosões. Basicamente, temos uma família de v.a.i.i.d $\left\{T_{n}\right\}_{n \in \mathbb{Z}_{+}}$. Uma partícula $n$ ao ser ativada assim permanece durante um tempo aleatório $T_{n}$ com distribuição contínua. Durante este tempo ela emite explosões nos instantes dados por um processo de Poisson. Isto é, dado $T_{n}=t$, o número de explosões emitidas pela partícula $n$ é Poisson $\left(\mu_{n} t\right)$, onde o parâmetro $\mu_{n}$ é o mesmo para toda partícula. O alcance da i-ésima explosão realizada pela partícula $n$ é dada pela variável aleatória $\overline{R_{n}^{i}}$.

Teorema 3.2.1. Suponha o processo Firework Contínuo em sua versão homogênea. Defina

$$
f_{\bar{R}, T, \mu}(n):=n\left\{1-M_{T}(-\mu \mathbb{P}(\bar{R} \geq n))\right\}
$$

Então:

Se $\lim _{n \rightarrow \infty} f_{\bar{R}, T, \mu}(n)<1$, então o processo morre quase certamente.

Se $\lim _{n \rightarrow \infty} f_{\bar{R}, T, \mu}(n)=1$ e existe $N$ tal que para todo $n \geq N$,

$$
M_{T}(-\mu \mathbb{P}(\bar{R} \geq n))>\frac{n-2}{n-1},
$$

então o processo morre quase certamente;

Se $\lim _{n \rightarrow \infty} f_{\bar{R}, T, \mu}(n)>1$, então o processo tem probabilidade positiva de sobreviver $e$ nesse caso,

$$
\mathbb{P}(V) \geq \prod_{n=0}^{\infty}\left[1-M_{T}\left(-\mu \sum_{j=0}^{n} \mathbb{P}(\bar{R} \geq j+1)\right)\right] .
$$

Prova da Proposição 3.2.1

Associamos cada realização do Processo a uma realização do Processo Firework com número aleatório $\mathrm{N}$ de tentativas de modo que

$$
\mathbb{P}(N=k)=\int_{0}^{\infty} \mathbb{P}(N=k \mid T=t) f(t) d t
$$


Como

$$
N \mid T=t \sim \text { Poisson }(\mu t)
$$

temos

$$
\mathbb{P}(N=k)=\int_{0}^{\infty} \frac{e^{-\mu t}(\mu t)^{k}}{k !} f(t) d t
$$

Multiplicando ambos lados da equação acima por $[P(\bar{R}<n)]^{k}$ e somando sobre k, obtemos

$$
\begin{aligned}
\mathbb{E}\left[\mathbb{P}(\bar{R}<n)^{N}\right] & =\sum_{k=0}^{\infty}[\mathbb{P}(\bar{R}<n)]^{k} \mathbb{P}(N=k)=\sum_{k=0}^{\infty}[\mathbb{P}(\bar{R}<n)]^{k} \int_{0}^{\infty} \frac{e^{-\mu t}(\mu t)^{k}}{k !} f(t) d t \\
& =\int_{0}^{\infty} \sum_{k=0}^{\infty}[\mathbb{P}(\bar{R}<n)]^{k} \frac{e^{-\mu t}(\mu t)^{k}}{k !} f(t) d t .
\end{aligned}
$$

Mas,

$$
\begin{aligned}
\int_{0}^{\infty} \sum_{k=0}^{\infty}[\mathbb{P}(\bar{R}<n)]^{k} \frac{e^{-\mu t}(\mu t)^{k}}{k !} f(t) d t & =\int_{0}^{\infty} \sum_{k=0}^{\infty} \frac{[\mathbb{P}(\bar{R}<n)(\mu t)]^{k}}{k !} e^{-\mu t} f(t) d t \\
& =\int_{0}^{\infty} e^{\mu t \mathbb{P}(\bar{R}<n)} e^{-\mu t} f(t) d t \\
& =\int_{0}^{\infty} e^{\mu t \mathbb{P}(\bar{R}<n)-\mu t} f(t) d t \\
& =\int_{0}^{\infty} e^{-\mu t \mathbb{P}(\bar{R} \geq n)} f(t) d t
\end{aligned}
$$

Logo, $\mathbb{E}\left[\mathbb{P}(\bar{R}<n)^{N}\right]=\mathbb{E}\left[e^{-\mu \mathbb{P}(\bar{R} \geq n) T}\right]$.

Daqui, a Proposição 3.1.0.1 nos dá o resultado.

Corolário 3.2.1.1. Seja o caso onde $T \sim \exp (\lambda)$.Então

Se $\lim _{n \rightarrow \infty} n \mathbb{P}(\bar{R} \geq n)>\frac{\lambda}{\mu}$ então o processo tem probabilidade positiva de sobreviver.

Nesse caso,

$$
\mathbb{P}(V) \geq \prod_{n=0}^{\infty}\left[1-\frac{\lambda^{n+1}}{\prod_{j=0}^{n}[\lambda+\mu \mathbb{P}(\bar{R} \geq j+1)]}\right]
$$


Se $\lim _{n \rightarrow \infty} n \mathbb{P}(\bar{R} \geq n)=\frac{\lambda}{\mu}$ e existe $N$ tal que $\forall n \geq N, \mathbb{P}(\bar{R} \geq n)<\frac{\lambda}{\mu(n-2)}$

então o processo morre quase certamente.

Se $\lim _{n \rightarrow \infty} n \mathbb{P}(\bar{R} \geq n)<\frac{\lambda}{\mu}$ então o processo morre quase certamente.

Prova do Corolário 3.2.1.1

Observe que se $\mathrm{T} \sim \exp (\lambda)$, então

$$
\mathbb{E}\left(c^{T}\right)=\frac{\lambda}{\lambda-\ln c}
$$

O restante segue do Teorema 3.2.1.

Exemplo 3.2.1.1. Considere um Processo Firework Contínuo sobre os inteiros não-negativos com alcances

$$
\mathbb{P}(R=k)=\frac{1}{(k+1)(k+2)}, k=0,1,2, \cdots,
$$

$\mu=4$ e $T \sim \operatorname{Exp}(2)$. Pelo Corolário 3.2.1.1, $\mathbb{P}(V) \geq \frac{1}{10}$.

Exemplo 3.2.1.2. Considere um Processo Firework Contínuo sobre os inteiros não-negativos com alcances

$$
\mathbb{P}(R=k)=\frac{1}{(k+1)(k+2)}, k=0,1,2, \cdots,
$$

$\mu=2$ e $T \sim \operatorname{Exp}(2)$. Pelo Corolário 3.2.1.1, $\mathbb{P}(V)=0$.

\subsection{O caso Heterogêneo}

No caso heterogêneo todos as variáveis envolvidas são independentes, entretanto pelo menos um dos conjuntos de variáveis (alcance das explosões ou tempo de ativação ou tempo entre explosões) é não identicamente distribuído ou as partículas não estão necessariamente sobre $\mathbb{Z}_{+}$. Basicamente, temos uma família de variáveis independentes $\left\{T_{n}\right\}_{n \in \mathbb{Z}_{+}}$, não necessaramente identicamente distribuídas. Uma partícula $n$ ao ser ativada assim permanece durante um tempo aleatório $T_{n}$ com distribuição contínua. Durante este tempo ela emite explosões nos instantes dados por um processo de Poisson. Isto é, dado $T_{n}=t$, o número de explosões emitidas pela partícula $n$ é Poisson $\left(\mu_{n} t\right)$, onde o parâmetro $\mu_{n}$ pode variar dependendo da partícula. $\mathrm{O}$ alcance da i-ésima explosão realizada pela partícula $n$ é dada pela variável aleatória $\overline{R_{n}^{i}}$. 
Teorema 3.3.1. Suponha o processo Firework Contínuo em sua versão não homogênea. Se as posições das partículas satisfazem $u_{n+1}-u_{n} \leq m e$

$$
\sum_{n=0}^{\infty} \prod_{j=0}^{n} M_{T_{n-j}}\left(-\mu_{n-j} \mathbb{P}\left(\bar{R}_{n-j} \geq(j+1) m\right)\right)<\infty
$$

o processo sobrevive com probabilidade positiva. Nesse caso,

$$
\mathbb{P}(V) \geq \prod_{n=0}^{\infty}\left[1-\prod_{j=0}^{n} M_{T_{n-j}}\left(-\mu_{n-j} \mathbb{P}\left(\bar{R}_{n-j} \geq(j+1) m\right)\right)\right]
$$

Por outro lado, se $\left|u_{n+1}-u_{n}\right| \geq k$ e as marginais do processo satisfazem:

1.

$$
M_{T_{i}}\left(-\mu_{i} \mathbb{P}\left(\bar{R}_{i} \geq k\right)\right) \in(0,1)
$$

2.

$$
\lim _{n \rightarrow \infty} \prod_{i=0}^{n} M_{T_{i}}\left(-\mu_{i} \mathbb{P}\left(\bar{R}_{i} \geq k(2 n-i)\right)\right)=1 ;
$$

3.

$$
\lim _{n \rightarrow \infty} \prod_{i=n}^{2 n-1} M_{T_{i}}\left(-\mu_{i} \mathbb{P}\left(\bar{R}_{i} \geq k(2 n-i)\right)\right)>0
$$

então o processo morre quase certamente.

Prova do Teorema 3.3.1

Assim como na prova do Teorema anterior, associamos cada realização do Processo a uma realização do Processo Firework com número aleatório $\mathrm{N}$ de tentativas de modo que

$$
\mathbb{P}\left(N_{i}=k\right)=\int_{0}^{\infty} \mathbb{P}\left(N_{i}=k \mid T_{i}=t\right) f(t) d t,
$$

o que nos leva a relação

$$
\mathbb{E}\left[\mathbb{P}\left(\bar{R}_{i}<n\right)^{N_{i}}\right]=\mathbb{E}\left[e^{-\mu_{i} \mathbb{P}\left(\bar{R}_{i} \geq n\right) T_{i}}\right] .
$$

O resultado segue da Proposição 3.1.0.2. 
Exemplo 3.3.1.1. Considere um Processo Firework Contínuo sobre os inteiros não-negativos com alcances

$$
\mathbb{P}(R=0)=1-p \text { e } \mathbb{P}(R=1)=p
$$

e suponha $T_{n} \sim \operatorname{Exp}\left(\lambda_{n}\right)$. Pelo Teorema 3.3 .1 o processo sobrevive com probabilidade positiva se

$$
\sum_{n=0}^{\infty} \frac{\lambda_{n}}{\lambda_{n}+p \mu_{n}}<\infty
$$

Além disso,

$$
\mathbb{P}(V)=\prod_{n=0}^{\infty} \frac{p \mu_{n}}{p \mu_{n}+\lambda_{n}} .
$$

Por outro lado, $\mathbb{P}(V)=0$ se

$$
\lim _{n \rightarrow \infty} \frac{\lambda_{n}}{\lambda_{n}+p \mu_{n}}>0 .
$$


36CAPÍTULO 3. PROCESSO FIREWORK CONTÍNUO UNIDIMENSIONAL 


\section{Capítulo 4}

\section{Processo Firework Discreto em Árvores}

Neste capítulo consideramos o processo Firework Discreto em árvores. Cada vértice da árvore possui exatamente uma partícula. Inicialmente, todas as partículas estão inativas, exceto a partícula sobre a raiz. Esta emite uma explosão de alcance aleatório. Todas as partículas atingidas pela explosão, emitem no próximo instante suas explosões e assim por diante. Aqui consideramos as explosões como variáveis aleatórias independentes e identicamente distribuídas discretas, assumindo valores nos inteiros não-negativos.

Seja $T=(\mathcal{V}, \mathcal{E})$ uma árvore qualquer e $O$ sua raiz. Associamos a cada $v \in \mathcal{V}$ um alcance de explosão $R_{v}$, isto é para o processo Firework Discreto na árvore, temos uma família de v.a.i.i.d. $\left\{R_{v}\right\}_{v \in \mathcal{V}}$ assumindo valores nos inteiros não-negativos. Desta forma apresentamos todos os resultados em função de uma variável aleatória $R$ de mesma distribuição que cada uma das variáveis $R_{v}$. Representamos a medida de probabilidade do processo por $\mathbb{P}$ e o evento "Processo Firework sobrevive" por $V$.

Para a árvore $T$, dados dois vértices $u$ e $v$ escrevemos $u \leq v$ se $u$ é um dos vértices do caminho entre $O$ e $v$. Nesse caso, dizemos que $v$ é descendente de $u$. Definimos o conjunto de descendentes de $u$ por

$$
T^{u}:=\{v \in \mathcal{V}: u \leq v\}
$$

e para todo $n \geq 1$ definimos

$$
T_{n}^{u}:=\left\{v \in T^{u}:|v| \leq|u|+n\right\}
$$

Escrevemos ainda

$$
M_{n}(u):=\left|\partial T_{n}^{u}\right|:=\left|\left\{v \in T^{u}:|v|=|u|+n\right\}\right|
$$


Definição 4.0.1.1. Para uma árvore $T$ definimos

$$
\operatorname{dim} \inf \partial T:=\lim _{n \rightarrow \infty} \min _{v \in \mathcal{V}} \frac{1}{n} \ln M_{n}(v) .
$$

Se $T=T_{d}$, isto é, se $T$ é a árvore homogênea de grau $d+1$, então temos $\operatorname{dim} \inf \partial T=\ln d$.

Lema 4.0.1.1. O limite dim inf $\partial T$ existe para toda árvore $T$.

Prova do Lema 4.0.1.1

Para qualquer $v \in \mathcal{V}$ e para todo $m, n \geq 1$ temos

$$
M_{m+n}(v) \geq M_{m}(v)\left(\min _{u \in \partial T_{m}^{v}} M_{n}(u)\right) \geq M_{m}(v)\left(\min _{u \in \mathcal{V}} M_{n}(u)\right) .
$$

Logo,

$$
M_{m+n}(v) \geq\left(\min _{v \in \mathcal{V}} M_{m}(v)\right)\left(\min _{v \in \mathcal{V}} M_{n}(v)\right) .
$$

Assim, $\ln \left[\min _{v \in \mathcal{V}} M_{n}(v)\right]$ é superaditiva e portanto, pelo Lema de Fekete, existe o limite

$$
\lim _{n \rightarrow \infty} \frac{1}{n} \ln \left[\min _{v \in \mathcal{V}} M_{n}(v)\right]=\sup _{n} \frac{1}{n} \ln \left[\min _{v \in \mathcal{V}} M_{n}(v)\right]=\lim _{n \rightarrow \infty} \min _{v \in \mathcal{V}} \frac{1}{n} \ln M_{n}(v)
$$

O leitor pode encontrar mais informações sobre dim inf $\partial T$ na seção 13.5 em [10], onde os autores Lyons e Peres apresentam não só esta, mas também outras constantes baseando-se em idéias não publicadas de Furstenberg.

\section{1 Árvore Homogênea}

Para o Processo Firework Discreto em Árvores Homogêneas apresentamos condições de sobrevivência e limitantes para a probabilidade de sobrevivência.

\subsubsection{Condições gerais para sobrevivência}

Teorema 4.1.1. Seja um processo Firework Discreto na árvore homogênea de grau $d+1, d \geq 2$, tal que

$$
\mathbb{P}(R=0) \notin\{0,1\} .
$$


Se

$$
\mathbb{E}\left(d^{R}\right)>1+\mathbb{P}(R=0),
$$

então o processo sobrevive com probabilidade positiva. Por outro lado, se

$$
\mathbb{E}\left(d^{R}\right) \leq 2-\frac{1}{d}
$$

então o processo morre quase certamente.

Prova do Teorema 4.1.1

Suponha

$$
\mathbb{E}\left(d^{R}\right)>1+\mathbb{P}(R=0) .
$$

Vamos definir um processo de ramificação $\left\{\mathcal{X}_{n}\right\}_{n \in \mathbb{Z}+} \operatorname{com} \mathcal{X}_{0}=1$ e com a distribuição do número de descendentes de um indivíduo satisfazendo :

$$
\begin{aligned}
& \mathbb{P}\left(X=d^{k}\right)=\mathbb{P}(R=k) \text { se } R \neq 0 \\
& \mathbb{P}(X=0)=\mathbb{P}(R=0) .
\end{aligned}
$$

Assim X toma valores no conjunto

$$
\left\{0, d, d^{2}, d^{3}, d^{4}, \cdots\right\} .
$$

Dado que uma partícula ativa teve explosão de alcance $k$, consideramos como descendentes no processo de ramificação apenas as partículas com distância $\mathrm{k}$ dela. Note que a sobrevivência do processo de ramificação implica na sobrevivência do processo Firework Discreto. Temos:

$$
\mathbb{E}(X)=\sum_{k=0}^{\infty} k \mathbb{P}(X=k)=\sum_{k=1}^{\infty} d^{k} \mathbb{P}\left(X=d^{k}\right)=\sum_{k=1}^{\infty} d^{k} \mathbb{P}(R=k) .
$$

Por outro lado,

$$
\mathbb{E}\left(d^{R}\right)=\sum_{k=0}^{\infty} d^{k} \mathbb{P}(R=k)
$$

Daí

$$
\mathbb{E}\left(d^{R}\right)=\mathbb{E}(X)+\mathbb{P}(R=0) .
$$

Por fim a condição

$$
\mathbb{E}\left(d^{R}\right)>1+\mathbb{P}(R=0)
$$

nos dá

$$
\mathbb{E}(X)>1
$$


donde o processo sobrevive com probabilidade positiva (veja Teorema 5.3.1). Agora consideremos o caso onde

$$
\mathbb{E}\left(d^{R}\right) \leq 2-\frac{1}{d}
$$

Seja $\left\{\mathcal{Y}_{n}\right\}_{n \in \mathbb{Z}+}$ um processo de ramificação tal que $\mathcal{Y}_{0}=1$ e cuja distribuição do número de descendentes de um indivíduo satisfaz

$$
\mathbb{P}\left(Y=\frac{d\left(d^{k}-1\right)}{d-1}\right)=\mathbb{P}(R=k) \text { para todo } k .
$$

Fixada uma partícula ativa, é como se todas as partículas ativadas por ela se movessem para a posição da partícula mais distante que foi ativada. E somente aí realizassem o processo de ativação de novas partículas. Note que aqui todas as partículas ativadas são descendentes no processo de ramificação. Neste caso, a morte do processo de ramificação implica na morte do processo Firework Discreto.

Temos:

$$
\begin{gathered}
\mathbb{E}(Y)=\sum_{k=0}^{\infty} k \mathbb{P}(Y=k)=\sum_{k=1}^{\infty} k \mathbb{P}(Y=k)=\sum_{k=1}^{\infty} \frac{d\left(d^{k}-1\right)}{d-1} \mathbb{P}\left(Y=\frac{d\left(d^{k}-1\right)}{d-1}\right) \\
=\sum_{k=1}^{\infty} \frac{d\left(d^{k}-1\right)}{d-1} \mathbb{P}(R=k)=\frac{d}{d-1}\left[\sum_{k=1}^{\infty} d^{k} \mathbb{P}(R=k)-\sum_{k=1}^{\infty} \mathbb{P}(R=k)\right] .
\end{gathered}
$$

Por outro lado

$$
\mathbb{E}\left(d^{R}\right)=\sum_{k=0}^{\infty} d^{k} \mathbb{P}(R=k)=\sum_{k=1}^{\infty} d^{k} \mathbb{P}(R=k)+\mathbb{P}(R=0) .
$$

Assim,

$$
\mathbb{E}(Y)=\frac{d}{d-1}\left[\mathbb{E}\left(d^{R}\right)-\mathbb{P}(R=0)-(1-\mathbb{P}(R=0))\right]=\frac{d}{d-1}\left[\mathbb{E}\left(d^{R}\right)-1\right] .
$$

Agora basta observar que

$$
\mathbb{E}(Y) \leq 1 \Longleftrightarrow \mathbb{E}\left(d^{R}\right) \leq 2-\frac{1}{d} .
$$

Assim, temos a prova para a segunda parte (veja Teorema 5.3.1). 
Corolário 4.1.1.1. Seja um processo Firework Discreto na árvore homogênea de grau $d+1 d \geq 2$, tal que

$$
\mathbb{P}(R=k)=(1-p) p^{k}, k=0,1,2, \cdots
$$

O processo tem probabilidade positiva de sobreviver se

$$
d p^{2}-2 d p+1<0
$$

Por outro lado, o processo morre quase certamente se $2 p d \leq 1$.

Assim, fixado d, o processo tem probabilidade positiva de sobreviver se

$$
p>1-\sqrt{1-\frac{1}{d}}
$$

e morre quase certamente se

$$
p \leq \frac{1}{2 d}
$$

Por outro lado, fixado p, o processo tem probabilidade positiva de sobreviver se

$$
d>\frac{1}{p(2-p)}
$$

e morre quase certamente se

$$
d \leq \frac{1}{2 p}
$$

Prova do Corolário 4.1.1.1

Observe que

$$
\mathbb{E}\left(d^{R}\right)=\sum_{k=0}^{\infty} d^{k}(1-p) p^{k}= \begin{cases}\infty, & \text { se } d p \geq 1 \\ \frac{1-p}{1-d p}, & d p<1\end{cases}
$$

A condição para morte quase certa implica $\frac{1-p}{1-d p} \leq 2-\frac{1}{d}$ que é equivalente a $p \leq \frac{1}{2 d}$. Por outro lado, a condição para chance positiva de sobrevivência nos dá $\frac{1-p}{1-d p}>2-p$ donde $d p^{2}-2 d p+1<0$ que é satisfeita quando $p>1-\sqrt{1-\frac{1}{d}}$.

Exemplo 4.1.1.1. Seja um processo Firework Discreto na árvore homogênea de grau 5 tal que

$$
\mathbb{P}(R=k)=(1-p) p^{k}, k=0,1,2, \cdots
$$

Temos $\mathbb{P}(V)>0$ se $p>\frac{2-\sqrt{3}}{2} \approx 0,1339$ e $\mathbb{P}(V)=0$ se $p<\frac{1}{8}=0,125$. 
Exemplo 4.1.1.2. Seja um processo Firework Discreto na árvore homogênea de grau 10 tal que

$$
\mathbb{P}(R=k)=(1-p) p^{k}, k=0,1,2, \cdots
$$

Temos $\mathbb{P}(V)>0$ se $p>\frac{3-2 \sqrt{2}}{3} \approx 0,05719$ e $\mathbb{P}(V)=0$ se $p<\frac{1}{18} \approx 0,05556$.

Corolário 4.1.1.2. Seja um processo Firework Discreto na árvore homogênea de grau $d+1, d \geq 2$, tal que

$$
\mathbb{P}(R=k)=\frac{e^{-\lambda} \lambda^{k}}{k !}, k=0,1,2, \cdots
$$

O processo tem probabilidade positiva de sobreviver se

$$
\left(e^{\lambda}\right)^{d}-e^{\lambda}-1>0
$$

e o processo morre quase certamente se

$$
\lambda(d-1) \leq \ln \frac{2 d-1}{d} .
$$

Fixado $\lambda$, o processo tem probabilidade positiva de sobreviver se

$$
d>\frac{1}{\lambda} \ln \left(1+e^{\lambda}\right)
$$

Fixado d, o processo morre quase certamente se

$$
\lambda \leq \ln \sqrt[d-1]{2-\frac{1}{d}}
$$

Prova do Corolário 4.1.1.2

Observe que

$$
\mathbb{E}\left(d^{R}\right)=\sum_{k=0}^{\infty} d^{k} \frac{e^{-\lambda} \lambda^{k}}{k !}=e^{-\lambda} \sum_{k=0}^{\infty} \frac{(d \lambda)^{k}}{k !}=e^{\lambda(d-1)} .
$$

A condição para morte quase certa implica $e^{\lambda(d-1)} \leq 2-\frac{1}{d}$ que é equivalente a $\lambda(d-1) \leq \ln \frac{2 d-1}{d}$. Por outro lado, a condição para chance positiva de sobrevivência nos dá $e^{\lambda(d-1)}>1+e^{-\lambda}$ que é equivalente a $\left(e^{\lambda}\right)^{d}-e^{\lambda}-1>0$. 
Corolário 4.1.1.3. Seja um processo Firework Discreto na árvore homogênea de grau $d+1 d \geq 2$, tal que

$$
\mathbb{P}(R=k)=\left(\begin{array}{l}
n \\
k
\end{array}\right) p^{k}(1-p)^{n-k}, k=0,1,2, \cdots, n .
$$

O processo tem probabilidade positiva de sobreviver se

$$
(p d+1-p)^{n}-(1-p)^{n}>1,
$$

e o processo morre quase certamente se

$$
d(p d+1-p)^{n} \leq 2 d-1
$$

Fixados p e n, o processo tem probabilidade positiva de sobreviver se

$$
d>\frac{1}{p}\left[\sqrt[n]{1+(1-p)^{n}}-1\right]+1
$$

Fixados d e n, o processo morre quase certamente se

$$
p \leq \frac{1}{(d-1)}\left[\sqrt[n]{\frac{2 d-1}{d}}-1\right]
$$

Prova do Corolário 4.1.1.3

Observe que

$\mathbb{E}\left(d^{R}\right)=\sum_{k=0}^{n} d^{k}\left(\begin{array}{l}n \\ k\end{array}\right) p^{k}(1-p)^{n-k}=\sum_{k=0}^{n}\left(\begin{array}{l}n \\ k\end{array}\right)(d p)^{k}(1-p)^{n-k}=(d p+1-p)^{n}$.

A condição para morte quase certa implica $(p d+1-p)^{n} \leq 2-\frac{1}{d}$ que é equivalente a $d(p d+1-p)^{n} \leq 2 d-1$. Por outro lado, a condição para chance positiva de sobrevivência nos dá $(d p+1-p)^{n}>1+(1-p)^{n}$ que é equivalente a $(p d+1-p)^{n}-(1-p)^{n}>1$.

\subsubsection{Limites para a Probabilidade de Sobrevivência}

Primeiro provaremos o seguinte Lema:

Lema 4.1.1.1. Considere o processo Firework Discreto numa árvore onde $M_{n}(v)=d^{n}$ para todo vértice $v$. Suponha $\mathbb{E}\left(d^{R}\right)>1+\mathbb{P}(R=0)$. Temos

$$
1-\rho \leq \mathbb{P}(V) \leq 1-\psi,
$$


onde $\rho$ e $\psi$ são soluções de

$$
\left\{\begin{array}{l}
\mathbb{E}\left(\rho^{d^{R}}\right)+(1-\rho) \mathbb{P}(R=0)=\rho \\
\mathbb{E}\left(\psi^{\frac{d}{d-1}\left(d^{R}-1\right)}\right)=\psi
\end{array}\right.
$$

Prova do Lema 4.1.1.1

Considere o processo de ramificação $\left\{\mathcal{X}_{n}\right\}_{n \in \mathbb{Z}+}$ definido na prova do Teorema 4.1.1. Temos $\mathbb{E}\left(d^{R}\right)=\mathbb{P}(R=0)+\mathbb{E}(X)$.

Observe que o processo Firework Discreto domina este processo de ramificação. Vamos então obter a probabilidade de extinção para esse processo de ramificação. Temos que $\rho=\varphi_{X}(\rho)$ onde $\rho$ é a probabilidade de extinção e $\varphi_{X}$ é a função geradora de probabilidade de $X$, isto é, $\varphi_{X}(s)=\mathbb{E}\left(s^{X}\right)$ (veja Teorema 5.3.1 no apêndice). Logo

$$
\begin{aligned}
\varphi_{X}(s) & =\mathbb{E}\left(s^{X}\right)=\sum_{k} s^{k} \mathbb{P}(X=k) \\
& =\sum_{k=1}^{\infty} s^{d^{k}} \mathbb{P}\left(X=d^{k}\right)+s^{0} \mathbb{P}(X=0) \\
& =\sum_{k=1}^{\infty} s^{d^{k}} \mathbb{P}\left(X=d^{k}\right)+\mathbb{P}(X=0) \\
& =\sum_{k=0}^{\infty} s^{d^{k}} \mathbb{P}\left(X=d^{k}\right)+\mathbb{P}(X=0)-s \mathbb{P}(R=0) \\
& =\mathbb{E}\left(s^{d^{R}}\right)+(1-s) \mathbb{P}(R=0) .
\end{aligned}
$$

Se $\rho=\varphi_{X}(\rho)$, então segue $\mathbb{E}\left(\rho^{d^{R}}\right)+(1-\rho) \mathbb{P}(R=0)=\rho$. Pela construção, $\mathbb{P}\left(V^{C}\right) \leq \rho$ donde $\mathbb{P}(V) \geq 1-\rho$.

Considere o processo de ramificação $\left\{\mathcal{Y}_{n}\right\}_{n \in \mathbb{Z}+}$ definido na prova do Teorema 4.1.1. Temos

$$
\mathbb{E}(Y)=\frac{d}{d-1}\left[\mathbb{E}\left(d^{R}\right)-1\right]
$$

Observe que o processo Firework Discreto é dominado por este processo de ramificação. Vamos então obter a probabilidade de extinção para esse processo de ramificação. Temos que $\psi=\varphi_{Y}(\psi)$ onde $\psi$ é a probabilidade de extinção e $\varphi_{Y}$ é a função geradora de probabilidade de $Y$, isto é, $\varphi_{Y}(s)=$ 
$\mathbb{E}\left(s^{Y}\right)$ (veja Teorema 5.3.1 no apêndice). Logo

$$
\begin{aligned}
\varphi_{Y}(s) & =\mathbb{E}\left(s^{Y}\right)=\sum_{k} s^{k} \mathbb{P}(Y=k) \\
& =\sum_{k=0}^{\infty} s^{\frac{d}{d-1}\left(d^{k}-1\right)} \mathbb{P}\left(Y=\frac{d}{d-1}\left(d^{k}-1\right)\right) \\
& =\sum_{k=0}^{\infty} s^{\frac{d}{d-1}\left(d^{k}-1\right)} \mathbb{P}(R=k) \\
& =\mathbb{E}\left(s^{\frac{d}{d-1}\left(d^{R}-1\right)}\right) .
\end{aligned}
$$

Se $\psi=\varphi_{Y}(\psi)$, então $\mathbb{E}\left(\psi^{\frac{d}{d-1}\left(d^{R}-1\right)}\right)=\psi$. Pela construção, $\mathbb{P}\left(V^{C}\right) \geq \psi$ donde $\mathbb{P}(V) \leq 1-\psi$.

Teorema 4.1.2. Considere o processo Firework Discreto na árvore homogênea de grau $d+1$. Sejam $\rho$ e $\psi$ satisfazendo

$$
\left\{\begin{array}{l}
\mathbb{E}\left(\rho^{d^{R}}\right)+(1-\rho) \mathbb{P}(R=0)=\rho \\
\mathbb{E}\left(\psi^{\frac{d}{d-1}\left(d^{R}-1\right)}\right)=\psi
\end{array}\right.
$$

Então

$$
1-\left(1-\rho^{\frac{d+1}{d}}\right) \mathbb{P}(R=0)-\mathbb{E}\left(\rho^{\frac{(d+1)}{d} d^{R}}\right) \leq \mathbb{P}(V) \leq 1-\mathbb{E}\left(\psi^{\frac{(d+1)}{d-1}\left(d^{R}-1\right)}\right) .
$$

Prova do Teorema 4.1.2

Seja $\lambda$ a probabilidade de sobrevivência do processo Firework Discreto numa árvore onde $M_{n}(v)=d^{n}$ para todo vértice $v$. Então, pelo Lema 4.1.1.1, $1-\rho \leq \lambda \leq 1-\psi$. Escreva

$$
V=V \bigcap\left[\bigcup_{k=0}^{\infty}\left\{R_{0}=k\right\}\right],
$$

onde $R_{0}$ é o alcance de explosão da partícula presente na raíz da árvore. Temos:

$$
\mathbb{P}(V)=\sum_{k=0}^{\infty} \mathbb{P}\left(V \mid R_{0}=k\right) \mathbb{P}\left(R_{0}=k\right) .
$$


Lema 4.1.2.1.

$$
\mathbb{P}\left(V \mid R_{0}=k\right) \geq 1-(1-\lambda)^{(d+1) d^{k-1}} \quad k=1,2, \cdots
$$

Prova do Lema 4.1.2.1

Consideraremos a explosão emitida pela partícula na raíz. Se $R_{0}=k$, temos $(d+1) d^{k-1}$ partículas ativadas e que distam $\mathrm{k}$ da raíz. Considere $(d+1) d^{k-1}$ cópias de árvores cujas raízes possuem cada uma, uma partícula ativada nesta primeira explosão. Considere um processo Firework Discreto em cada uma dessas árvores. Nestes termos, defina os eventos: $V_{k, i}$ : "Processo Firework Discreto relativo a i-ésima árvore sobrevive". Observe que cada uma destas árvores tem a propriedade $M_{n}(v)=d^{n}$ para todo vértice $v$. Assim, $\mathbb{P}\left(V_{k, i}\right)=\lambda$. Logo

$$
\begin{aligned}
\mathbb{P}\left(V \mid R_{0}=k\right) & \geq \mathbb{P}\left(\bigcup_{i=1}^{(d+1) d^{R_{0}-1}} V_{R_{0}, i} \mid R_{0}=k\right) \\
& =\mathbb{P}\left(\bigcup_{i=1}^{(d+1) d^{k-1}} V_{k, i} \mid R_{0}=k\right) \\
& =\mathbb{P}\left(\bigcup_{i=1}^{(d+1) d^{k-1}} V_{k, i}\right) \\
& =1-\mathbb{P}\left(\bigcap_{i=1}^{(d+1) d^{k-1}} V_{k, i}^{C}\right) \\
& =1-\prod_{i=1}^{(d+1) d^{k-1}} \mathbb{P}\left(V_{k, i}^{C}\right) \\
& =1-\prod_{i=1}^{(d+1) d^{k-1}}(1-\lambda) \\
& =1-(1-\lambda)^{(d+1) d^{k-1}} .
\end{aligned}
$$

Lema 4.1.2.2.

$$
\mathbb{P}\left(V \mid R_{0}=k\right) \leq 1-(1-\lambda)^{\frac{(d+1)}{d-1}\left[d^{k}-1\right]} \quad k=0,1, \cdots
$$


Prova do Lema 4.1.2.2

Se $R_{0}=k$, temos $\frac{(d+1)\left(d^{k}-1\right)}{d-1}$ partículas ativadas após a emissão de explosão da partícula na raíz. Considere $\frac{(d+1)\left(d^{k}-1\right)}{d-1}$ cópias de árvores cujas raízes possuem cada uma, uma partícula ativada nesta primeira explosão. Considere um processo Firework Discreto em cada uma dessas árvores. A idéia é que quando uma destas árvores esta imersa em outra, consideraremos duas árvores distintas com dois processos Fireworks Discreto independentes. Nestes termos, defina os eventos: $V_{k, i}$ : "Processo Firework Discreto relativo a i-ésima árvore sobrevive". Observe que cada uma destas árvores tem a propriedade $M_{n}(v)=d^{n}$ para todo vértice $v$. Assim, $\mathbb{P}\left(V_{k, i}\right)=\lambda$. Logo

$$
\begin{aligned}
\mathbb{P}\left(V \mid R_{0}=k\right) & \leq \mathbb{P}\left(\bigcup_{i=1}^{\frac{(d+1)}{d-1}\left[d^{\left.R_{0}-1\right]}\right.} V_{R_{0}, i} \mid R_{0}=k\right) \\
& =\mathbb{P}\left(\bigcup_{i=1}^{\frac{(d+1)}{d-1}\left[d^{k}-1\right]} V_{k, i} \mid R_{0}=k\right) \\
& =\mathbb{P}\left(\bigcup_{i=1}^{\frac{(d+1)}{d-1}\left[d^{k}-1\right]} V_{k, i}\right) \\
& =1-\mathbb{P}\left(\bigcap_{i=1}^{\frac{(d+1)}{d-1}\left[d^{k}-1\right]} V_{k, i}^{C}\right) \\
& =1-\prod_{i=1}^{\frac{(d+1)}{d-1}\left[d^{k}-1\right]} \mathbb{P}\left(V_{k, i}^{C}\right) \\
& =1-\prod_{i=1}^{\frac{(d+1)}{d-1}\left[d^{k}-1\right]}(1-\lambda) \\
& =1-(1-\lambda)^{\frac{(d+1)}{d-1}\left[d^{k}-1\right]} .
\end{aligned}
$$


Voltemos a prova do Teorema. Do Lema 4.1.2.1 segue

$$
\begin{aligned}
\mathbb{P}(V) & =\sum_{k=0}^{\infty} \mathbb{P}\left(V \mid R_{0}=k\right) \mathbb{P}\left(R_{0}=k\right) \geq \sum_{k=1}^{\infty}\left[1-(1-\lambda)^{(d+1) d^{k-1}}\right] \mathbb{P}(R=k) \\
& \geq \sum_{k=1}^{\infty}\left[1-\rho^{(d+1) d^{k-1}}\right] \mathbb{P}(R=k)=1-\mathbb{P}(R=0)-\sum_{k=1}^{\infty}\left[\rho^{\frac{(d+1)}{d} d^{k}}\right] \mathbb{P}(R=k) \\
& =1-\mathbb{P}(R=0)-\mathbb{E}\left(\rho^{\frac{(d+1)}{d} d^{R}}\right)+\rho^{\frac{(d+1)}{d}} \mathbb{P}(R=0) \\
& =1-\left(1-\rho^{\frac{d+1}{d}}\right) \mathbb{P}(R=0)-\mathbb{E}\left(\rho^{\frac{(d+1)}{d} d^{R}}\right) .
\end{aligned}
$$

Por outro lado, do Lema 4.1.2.2 segue

$$
\begin{aligned}
\mathbb{P}(V) & =\sum_{k=0}^{\infty} \mathbb{P}\left(V \mid R_{0}=k\right) \mathbb{P}\left(R_{0}=k\right) \leq \sum_{k=0}^{\infty}\left[1-(1-\lambda)^{\frac{(d+1)}{d-1}\left[d^{k}-1\right]}\right] \mathbb{P}(R=k) \\
& \leq \sum_{k=0}^{\infty}\left[1-\psi^{\frac{(d+1)}{d-1}\left[d^{k}-1\right]}\right] \mathbb{P}(R=k)=1-\sum_{k=0}^{\infty}\left[\psi^{\frac{(d+1)}{d-1}\left[d^{k}-1\right]}\right] \mathbb{P}(R=k) \\
& =1-\mathbb{E}\left(\psi^{\frac{(d+1)}{d-1}\left(d^{R}-1\right)}\right) .
\end{aligned}
$$

Exemplo 4.1.2.1. Considere o caso onde $d=2$ e $R \sim \operatorname{Binomial~}\left(4, \frac{1}{2}\right)$. Então $\rho$ e $\psi$ são, respectivamente, soluções de

$$
\left\{\begin{array}{l}
x^{16}+4 x^{8}+6 x^{4}+4 x^{2}-16 x+1=0 \\
x^{30}+4 x^{14}+6 x^{6}+4 x^{2}-16 x+1=0 .
\end{array}\right.
$$

Logo $\rho=0,0635146$ e $\psi=0,06350850$ e portanto, $0,937435919 \leq \mathbb{P}(V) \leq$ 0,937435962 .

Exemplo 4.1.2.2. Considere o caso onde $d=4$ e $R \sim \operatorname{Binomial~}\left(4, \frac{1}{4}\right)$. Então $\rho$ e $\psi$ são, respectivamente, soluções de

$$
\left\{\begin{array}{l}
x^{256}+12 x^{64}+54 x^{16}+108 x^{4}-256 x+81=0 \\
x^{340}+12 x^{84}+54 x^{20}+108 x^{4}-256 x+81=0 .
\end{array}\right.
$$

Logo $\rho=0,3208787235$ e $\psi=0,3208787200$ e portanto, $\mathbb{P}(V) \approx 0,682158629$.

Corolário 4.1.2.1. Considere o caso onde $\mathbb{P}(R=0)=1-p$ e $\mathbb{P}(R=1)=p$. Então

onde $\psi$ é solução de

$$
\mathbb{P}(V)=p\left(1-\psi^{d+1}\right)
$$

$$
p \psi^{d}-\psi+1-p=0 .
$$


Prova do Corolário 4.1.2.1.

Segue do Teorema 4.1.2.

Exemplo 4.1.2.3. Tome $\mathbb{P}(R=0)=1-p$ e $\mathbb{P}(R=1)=p, d=2$ e $p>\frac{1}{2}$. Temos:

$$
\mathbb{P}(V)=\frac{(2 p-1)\left(p^{2}-p+1\right)}{p^{2}} .
$$

Em particular, se $p=\frac{6}{10}, \mathbb{P}(V)=\frac{19}{45}$ e se $p=\frac{8}{10}, \mathbb{P}(V)=\frac{63}{80}$.

Exemplo 4.1.2.4. Tome $\mathbb{P}(R=0)=1-p$ e $\mathbb{P}(R=1)=p, d=3$ e $p>\frac{1}{3}$. Temos:

$$
\mathbb{P}(V)=\frac{3 p^{2}-2}{2 p}+\frac{(2-p)}{2} \sqrt{\frac{4}{p}-3} .
$$

Em particular, se $p=\frac{4}{10}, \mathbb{P}(V)=0,2166$, se $p=\frac{5}{10}, \mathbb{P}(V)=0,427$, se $p=\frac{8}{10}, \mathbb{P}(V)=0,7985$ e se $p=\frac{9}{10}, \mathbb{P}(V)=0,8999$.

Exemplo 4.1.2.5. Tome $\mathbb{P}(R=0)=\frac{4118}{6305}$ e $\mathbb{P}(R=1)=\frac{2187}{6305}$ e $d=8$. Temos:

$$
\mathbb{P}(V)=\frac{19171}{56745} \approx 0,337844
$$

\section{2 Árvore k-periódica}

Seja um conjunto de $\mathrm{k}$ números inteiros $d_{1}, d_{2}, \cdots, d_{k}$, tais que $d_{i} \geq 2$ para todo $i=1,2, \cdots k$. Chamaremos de árvore k-periódica aquela onde todo vértice que dista $n k+i-1$ da raíz tem grau $d_{i}+1$ para todo $i=$ $1,2, \cdots k$ e $n$ inteiro não negativo. Se $d_{1} \leq d_{2} \leq \cdots \leq d_{k}$, temos uma árvore k-periódica de graus crescentes. Por outro lado, se $d_{1} \geq d_{2} \geq \cdots \geq d_{k}$, temos uma árvore k-periódica de graus decrescentes.

Se desconsideramos na árvore k-periódica um dos ramos que partem da raiz, isto é, considerarmos a raiz com grau $d_{1}$ e não com grau $d_{1}+1$, teremos uma árvore quase $k$-periódica.

Considere uma árvore k-periódica com graus $d_{1}+1, d_{2}+1, \cdots, d_{k}+1$, $d_{i} \geq 2$ para todo $i=1,2, \cdots k$. Apresentaremos a seguir algumas quantidades úteis para a prova dos resultados desta seção.

Definição 4.2.0.1. Definimos a quantidade

$$
d_{(i)}:=\text { o i-ésimo menor dos } d_{i}
$$


Definição 4.2.0.2. Definimos as variáveis aleatórias indicadoras

$$
I_{i}:= \begin{cases}1, & \text { se } \mathrm{R}=\mathrm{kn}+\mathrm{i} \text { para algum } \mathrm{n} \text { inteiro não negativo; } \\ 0, & \text { caso contrário. }\end{cases}
$$

Definição 4.2.0.3. Definimos a média geométrica dos valores $d_{1}, d_{2}, \cdots, d_{k}$

$$
G:=\sqrt[k]{\prod_{j=1}^{k}} d_{j}
$$

Definição 4.2.0.4. Definimos as quantidades

$$
c_{0}:=1 \text { e } c_{i}:=\frac{\prod_{j=1}^{i} d_{(j)}}{\sqrt[k]{\prod_{j=1}^{k}\left(d_{j}\right)^{i}}}=\frac{\prod_{j=1}^{i} d_{(j)}}{G^{i}}, i=1, \cdots, k-1 .
$$

$\mathrm{O}$ valor $c_{i}$ é o quociente entre o produto dos $i$ menores valores no conjunto $\left\{d_{1}, d_{2}, \cdots, d_{k}\right\}$ pela $i$-ésima potência da média geométrica entre eles.

Definição 4.2.0.5. Definimos as quantidades

$$
\overline{c_{0}}:=1 \text { e } \overline{c_{i}}:=\frac{\prod_{j=1}^{i} d_{(k+1-j)}}{\sqrt[k]{\prod_{j=1}^{k}\left(d_{j}\right)^{i}}}=\frac{\prod_{j=1}^{i} d_{(k+1-j)}}{G^{i}}, i=1, \cdots, k-1 .
$$

$\mathrm{O}$ valor $\bar{c}_{i}$ é o quociente entre o produto dos $i$ maiores valores no conjunto $\left\{d_{1}, d_{2}, \cdots, d_{k}\right\}$ pela $i$-ésima potência da média geométrica entre eles.

Definição 4.2.0.6. Definimos as quantidades

$$
x_{n, i}:=\prod_{j=1}^{k}\left(d_{j}\right)^{n} \prod_{j=1}^{i} d_{(j)} . \text { Em particular, } x_{n, 0}:=\prod_{j=1}^{k}\left(d_{j}\right)^{n} .
$$

Considere o Processo Firework Discreto na árvore k-periódica de graus crescentes $d_{(1)}+1, d_{(2)}+1, \cdots, d_{(k)}+1$. Seja $v \neq O$ um vértice de grau $d_{(1)}+1$. Se a partícula deste vértice causa uma explosão de alcance $n k+i, x_{n, i}$ é o numero de partículas atingidas por esta explosão e que distam $n k+i$ de $v$.

Lema 4.2.0.3. Considere uma árvore $k$-periódica com graus $d_{1}+1, d_{2}+$ $1, \cdots, d_{k}+1, d_{i} \geq 2$ para todo $i=1,2, \cdots k$. Suponha que uma partícula num vértice $v \neq O$ é atingida e emite uma explosão de raio $R_{v}=n k+i$. Seja $s_{n, i}$ o numero de partículas atingidas por esta explosão e que distam $n k+i$ de $v$. Então

$$
s_{n, i} \geq x_{n, i}
$$


Prova do Lema 4.2.0.3

Basta observar que com os valores $d_{1}, d_{2}, \cdots, d_{k}$ é possível obter $k$ ! diferentes árvores quase $k$-periódicas. Se olharmos para a j-ésima geração de cada uma delas, a árvore que terá menos descendentes nessa geração é aquela cujos graus são, respectivamente, $d_{(1)}+1, d_{(2)}+1, \cdots, d_{(k)}+1$.

Definição 4.2.0.7. Definimos as quantidades

$$
\bar{x}_{n, i}:=\prod_{j=1}^{k}\left(d_{j}\right)^{n} \prod_{j=1}^{i} d_{(k+1-j)} . \text { Em particular, } \bar{x}_{n, 0}:=\prod_{j=1}^{k}\left(d_{j}\right)^{n} .
$$

Considere o Processo Firework Discreto na árvore k-periódica de graus decrescentes $d_{(k)}+1, d_{(k-1)}+1, \cdots, d_{(1)}+1$. Seja $v \neq O$ um vértice de grau $d_{(k)}+1$. Se a partícula deste vértice causa uma explosão de alcance $n k+i$, $\bar{x}_{n, i}$ é o numero de partículas atingidas por esta explosão e que distam $n k+i$ de $v$.

Lema 4.2.0.4. Considere uma árvore k-periódica com graus $d_{1}+1, d_{2}+$ $1, \cdots, d_{k}+1, d_{i} \geq 2$ para todo $i=1,2, \cdots k$. Suponha que uma partícula num vértice $v \neq O$ é atingida e emite uma explosão de raio $R_{v}=n k+i$. Seja $\bar{s}_{n, i}$ o numero de partículas atingidas por esta explosão e que distam $n k+i$ de v. Então

$$
\bar{s}_{n, i} \leq \bar{x}_{n, i}
$$

Prova do Lema 4.2.0.4

Basta observar que com os valores $d_{1}, d_{2}, \cdots, d_{k}$ é possível obter $k$ ! diferentes árvores quase $k$-periódicas. Se olharmos para a j-ésima geração de cada uma delas, a árvore que terá mais descendentes nessa geração é aquela cujos graus são, respectivamente, $d_{(k)}+1, d_{(k-1)}+1, \cdots, d_{(1)}+1$.

Definição 4.2.0.8. Definimos as quantidades

$$
y_{n, i}:=\frac{\left(d_{(1)}\right)^{n k+i}-1}{\left(d_{(1)}\right)^{n k+i-1}\left[d_{(1)}-1\right]} . \text { Em particular, } y_{0,0}:=0 .
$$

Lema 4.2.0.5. Considere uma árvore $k$-periódica com graus $d_{1}+1, d_{2}+$ $1, \cdots, d_{k}+1, d_{i} \geq 2$ para todo $i=1,2, \cdots k$. Suponha que uma partícula num vértice $v \neq O$ é atingida e emite uma explosão de raio $R_{v}=n k+i$. Seja $t_{n, i}$ o numero de partículas atingidas por esta explosão. Então

$$
t_{n, i} \leq\left\lfloor y_{n, i} \bar{x}_{n, i}\right\rfloor .
$$




\section{CAPÍTULO 4. PROCESSO FIREWORK DISCRETO EM ÁRVORES}

Prova do Lema 4.2.0.5

Do Lema 4.2.0.4 temos que o numero de partículas atingidas por esta explosão e que distam $n k+i$ de $v$ é no máximo $\bar{x}_{n, i}$. Logo

$t_{n, i} \leq\left\lfloor\bar{x}_{n, i}+\frac{\bar{x}_{n, i}}{d_{(1)}}+\frac{\bar{x}_{n, i}}{\left(d_{(1)}\right)^{2}}+\cdots+\frac{\bar{x}_{n, i}}{\left(d_{(1)}\right)^{n k+i-1}}\right\rfloor=\left\lfloor\frac{\left(d_{(1)}\right)^{n k+i}-1}{\left(d_{(1)}\right)^{n k+i-1}\left[d_{(1)}-1\right]} \bar{x}_{n, i}\right\rfloor$.

\subsubsection{Condições gerais para sobrevivência}

Teorema 4.2.1. Seja um processo Firework Discreto na árvore k-periódica com graus $d_{1}+1, d_{2}+1, \cdots, d_{k}+1, d_{i} \geq 2$, tal que

$$
\mathbb{P}(R=0) \notin\{0,1\} \text {. }
$$

Se

$$
\sum_{i=0}^{k-1} c_{i} \mathbb{E}\left[G^{R} I_{i}\right]>1+\mathbb{P}(R=0),
$$

então o processo sobrevive com probabilidade positiva. Se

$$
\sum_{i=0}^{k-1} \bar{c}_{i} \mathbb{E}\left[\left(1-\frac{1}{\left(d_{(1)}\right)^{R}}\right) G^{R} I_{i}\right] \leq 1-\frac{1}{d_{(1)}},
$$

então o processo morre quase certamente.

Prova do Teorema 4.2.1

Na prova desconsideramos um dos ramos que partem da raiz. Isto é, consideramos a raiz com grau $d_{1}$ e não com grau $d_{1}+1$. Chamaremos essa árvore de quase $k$ periódica. As condições de sobrevivência não se alteram.

Suponha

$$
\sum_{i=0}^{k-1} c_{i} \mathbb{E}\left[G^{R} I_{i}\right]>1+\mathbb{P}(R=0) .
$$

Vamos definir um processo de ramificação $\left\{\mathcal{X}_{n}\right\}_{n \in \mathbb{Z}+} \operatorname{com} \mathcal{X}_{0}=1$ e com a distribuição do número de descendentes de um indivíduo satisfazendo:

$$
\begin{aligned}
& \mathbb{P}\left(X=x_{n, i}\right)=\mathbb{P}(R=n k+i) \text { se } n k+i \neq 0 \text { e } \\
& \mathbb{P}(X=0)=\mathbb{P}(R=0) .
\end{aligned}
$$

Assim, X toma valores no conjunto $\left\{0, d_{(1)}, d_{(1)} d_{(2)}, d_{(1)} d_{(2)} d_{(3)} \cdots, d_{(1)} d_{(2)} \ldots d_{(k)}\right.$, $\left.d_{(1)} d_{(2)} \ldots d_{(k)} d_{(1)}, \cdots\left(d_{(1)} \ldots d_{(k)}\right)^{2},\left(d_{(1)} \ldots d_{(k)}\right)^{2} . d_{(1)}, \cdots\right\}$. Do Lema 4.2.0.3 segue 
que a sobrevivência do processo de ramificação assim descrito implica na sobrevivência do processo Firework Discreto.

Temos:

$$
\begin{aligned}
\mathbb{E}(X) & =\sum_{n=1}^{\infty} x_{n, 0} \mathbb{P}\left(X=x_{n, 0}\right)+\sum_{i=1}^{k-1} \sum_{n=0}^{\infty} x_{n, i} \mathbb{P}\left(X=x_{n, i}\right) \\
& =\sum_{n=1}^{\infty} x_{n, 0} \mathbb{P}(R=n k)+\sum_{i=1}^{k-1} \sum_{n=0}^{\infty} x_{n, i} \mathbb{P}(R=n k+i) \\
= & \sum_{i=0}^{k-1} c_{i} \sum_{n=0}^{\infty} \prod_{j=1}^{k}\left(\sqrt[k]{d_{j}}\right)^{n k+i} \mathbb{P}(R=n k+i)-\mathbb{P}(R=0) \\
= & \mathbb{E}\left[G^{R} I_{0}\right]+\sum_{i=1}^{k-1} c_{i} \mathbb{E}\left[G^{R} I_{i}\right]-\mathbb{P}(R=0) \\
= & \sum_{i=0}^{k-1} c_{i} \mathbb{E}\left[G^{R} I_{i}\right]-\mathbb{P}(R=0) .
\end{aligned}
$$

Por fim a condição

$$
\sum_{i=0}^{k-1} c_{i} \mathbb{E}\left[G^{R} I_{i}\right]>1+\mathbb{P}(R=0)
$$

nos dá

$$
\mathbb{E}(X)>1,
$$

donde o processo sobrevive com probabilidade positiva (veja Teorema 5.3.1). Agora consideremos o caso onde

$$
\sum_{i=0}^{k-1} c_{i} \mathbb{E}\left[\left(1-\frac{1}{\left(d_{(1)}\right)^{R}}\right) G^{R} I_{i}\right] \leq 1-\frac{1}{d_{(1)}}
$$

Seja $\left\{\mathcal{Y}_{n}\right\}_{n \in \mathbb{Z}+}$ um processo de ramificação tal que $\mathcal{Y}_{0}=1$ e cuja distribuição do número de descendentes de um indivíduo satisfaz

$$
\mathbb{P}\left(Y=\left\lfloor y_{n, i} \bar{x}_{n, i}\right\rfloor\right)=\mathbb{P}(R=n k+i), i=0,1,2, \cdots, k-1 .
$$

Do Lema 4.2.0.5 segue que a não sobrevivência do processo de ramificação descrito implica na não sobrevivência do processo Firework Discreto. 


$$
\begin{aligned}
\mathbb{E}(X) \leq & \left.\sum_{n=1}^{\infty} y_{n, 0} \bar{x}_{n, 0} \mathbb{P}\left(X=\left\lfloor y_{n, 0} \bar{x}_{n, 0}\right\rfloor\right\}\right)+\sum_{i=1}^{k-1} \sum_{n=0}^{\infty} y_{n, i} \bar{x}_{n, i} \mathbb{P}\left(X=\left\lfloor y_{n, i} \bar{x}_{n, i}\right\rfloor\right) \\
= & \sum_{n=1}^{\infty} y_{n, 0} \bar{x}_{n, 0} \mathbb{P}(R=n k)+\sum_{i=1}^{k-1} \sum_{n=0}^{\infty} y_{n, i} \bar{x}_{n, i} \mathbb{P}(R=n k+i) \\
= & \frac{d_{(1)}}{\left[d_{(1)}-1\right]} \sum_{n=1}^{\infty} \frac{\left(d_{(1)}\right)^{n k}-1}{\left(d_{(1)}\right)^{n k}} \bar{x}_{n, 0} \mathbb{P}(R=n k)+ \\
& \frac{d_{(1)}}{\left[d_{(1)}-1\right]} \sum_{i=1}^{k-1} \sum_{n=0}^{\infty} \frac{\left(d_{(1)}\right)^{n k+i}-1}{\left(d_{(1)}\right)^{n k+i}} \bar{x}_{n, i} \mathbb{P}(R=n k+i) \\
= & \frac{d_{(1)}}{\left[d_{(1)}-1\right]} \sum_{n=1}^{\infty} \frac{\left(d_{(1)}\right)^{n k}-1}{\left(d_{(1)}\right)^{n k}} \prod_{j=1}^{k}\left(\sqrt[k]{d_{j}}\right)^{n k} \mathbb{P}(R=n k)+ \\
& \frac{d_{(1)}}{\left[d_{(1)}-1\right]} \sum_{i=1}^{k-1} \bar{c}_{i} \sum_{n=0}^{\infty} \frac{\left(d_{(1)}\right)^{n k+i}-1}{\left(d_{(1)}\right)^{n k+i}} \prod_{j=1}^{k}\left(\sqrt[k]{d_{j}}\right)^{n k+i} \mathbb{P}(R=n k+i) \\
= & \frac{d_{(1)}}{\left[d_{(1)}-1\right]} \mathbb{E}\left[\left(1-\frac{1}{\left(d_{(1)}\right)^{R}}\right)^{G^{R}} I_{0}\right]+\sum_{i=1}^{k-1} \bar{c}_{i} \mathbb{E}\left[\left(1-\frac{1}{\left(d_{(1)}\right)^{R}}\right) G^{R} I_{i}\right] \\
= & \frac{d_{(1)}}{\left[d_{(1)}-1\right]} \sum_{i=0}^{k-1} \overline{c_{i}} \mathbb{E}\left[\left(1-\frac{1}{\left(d_{(1)}\right)^{R}}\right) G^{R} I_{i}\right] .
\end{aligned}
$$

Por fim, a condição

$$
\sum_{i=0}^{k-1} \overline{c_{i}} \mathbb{E}\left[\left(1-\frac{1}{\left(d_{(1)}\right)^{R}}\right) G^{R} I_{i}\right] \leq 1-\frac{1}{d_{(1)}}
$$

nos dá

$$
\mathbb{E}(X) \leq 1,
$$

donde o processo morre quase certamente (veja Teorema 5.3.1).

Exemplo 4.2.1.1. Seja um processo Firework Discreto na árvore periódica com $d_{1}=4$ e $d_{2}=9$ tal que

$$
\mathbb{P}(R=j)=(1-p) p^{j}, j=0,1,2, \cdots
$$

Temos $\mathbb{P}(V)>0$ se $36 p^{3}-68 p^{2}-4 p+1<0$. Para tal, basta tomar $p>0,0973$. Por outro lado, $\mathbb{P}(V)=0$ se $\frac{(1-p)(9 p+1)}{(1-6 p)(1+6 p)}-\frac{(1-p)(9 p+4)}{(2-3 p)(2+3 p)} \leq \frac{3}{4}$. Para tal, basta tomar $p \leq 0,0695$. 


\subsubsection{Limites para a Probabilidade de Sobrevivência}

Primeiro provaremos o seguinte Lema:

Lema 4.2.1.1. Considere o processo Firework Discreto numa árvore quase periódica de graus $d_{1}+1, d_{2}+1, \cdots, d_{k}+1, d_{i} \geq 2$ para todo $i=1,2, \cdots k$. Suponha

$$
\sum_{i=0}^{k-1} c_{i} \mathbb{E}\left[G^{R} I_{i}\right]>1+\mathbb{P}(R=0) .
$$

Temos

$$
1-\rho \leq \mathbb{P}(V) \leq 1-\psi,
$$

onde $\rho$ e $\psi$ são soluções de

$$
\begin{aligned}
& \sum_{i=0}^{k-1} \mathbb{E}\left[\rho^{c_{i} G^{R}} I_{i}\right]+(1-\rho) \mathbb{P}(R=0)=\rho \\
& \sum_{i=0}^{k-1} \mathbb{E}\left[\psi \psi^{\left\lfloor\bar{c}_{i} h(R)\right\rfloor} I_{i}\right]=\psi
\end{aligned}
$$

$\operatorname{com} h(R)=\frac{\left[d_{(1)}^{R}-1\right]}{d_{(1)}^{R-1}\left[d_{(1)}-1\right]} G^{R}$.

Prova do Lema 4.2.1.1

Considere o processo de ramificação $\left\{\mathcal{X}_{n}\right\}_{n \in \mathbb{Z}+}$ definido na prova do Teorema 4.2.1. Observe que o processo Firework Discreto domina este processo de ramificação. Vamos então obter a probabilidade de extinção para esse processo de ramificação. Temos que $\rho=\varphi_{X}(\rho)$ onde $\rho$ é a probabilidade de extinção e $\varphi_{X}$ é a função geradora de probabilidade de $X$, isto é, 
$\varphi_{X}(s)=\mathbb{E}\left(s^{X}\right)$ (veja Teorema 5.3.1 no apêndice). Logo

$$
\begin{aligned}
\varphi_{X}(s)= & \mathbb{E}\left(s^{X}\right)=\sum_{k} s^{k} \mathbb{P}(X=k)=s^{0} \mathbb{P}(X=0)+\sum_{n=1}^{\infty} s^{x_{n, 0}} \mathbb{P}\left(X=x_{n, 0}\right)+ \\
& \sum_{i=1}^{k-1} \sum_{n=0}^{\infty} s^{x_{n, i}} \mathbb{P}\left(X=x_{n, i}\right)=\mathbb{P}(R=0)+\sum_{n=1}^{\infty} s^{x_{n, 0}} \mathbb{P}(R=n k)+ \\
& \sum_{i=1}^{k-1} \sum_{n=0}^{\infty} s^{x_{n, i}} \mathbb{P}(R=n k+i)=\mathbb{P}(R=0)+\sum_{n=1}^{\infty} s^{G^{n k}} \mathbb{P}(R=n k)+ \\
& \sum_{i=1}^{k-1} \sum_{n=0}^{\infty} s^{c_{i} G^{n k+i}} \mathbb{P}(R=n k+i)=\mathbb{P}(R=0)-s \mathbb{P}(R=0)+ \\
& \sum_{i=0}^{k-1} \sum_{n=0}^{\infty} s^{c_{i} G^{n k+i}} \mathbb{P}(R=n k+i)=(1-s) \mathbb{P}(R=0)+\sum_{i=0}^{k-1} \mathbb{E}\left[s^{c_{i} g^{R}} I_{i}\right] .
\end{aligned}
$$

Se $\rho=\varphi_{X}(\rho)$, então segue $\sum_{i=0}^{k-1} \mathbb{E}\left[\rho^{c_{i} G^{R}} I_{i}\right]+(1-\rho) \mathbb{P}(R=0)=\rho$. Pela construção, $\mathbb{P}\left(V^{C}\right) \leq \rho$ donde $\mathbb{P}(V) \geq 1-\rho$.

Por outro lado, considere o processo de ramificação $\left\{\mathcal{Y}_{n}\right\}_{n \in \mathbb{Z}+}$ definido na prova do Teorema 4.2.1. Observe que o processo Firework Discreto é dominado por este processo de ramificação. Vamos então obter a probabilidade de extinção para esse processo de ramificação. Temos que $\psi=\varphi_{Y}(\psi)$ onde $\psi$ é a probabilidade de extinção e $\varphi_{Y}$ é a função geradora de probabilidade de $Y$, isto é, $\varphi_{Y}(s)=\mathbb{E}\left(s^{Y}\right)$ (veja Teorema 5.3.1 no apêndice). Logo

$$
\begin{aligned}
\varphi_{Y}(s)= & \mathbb{E}\left(s^{Y}\right)=\sum_{k} s^{k} \mathbb{P}(Y=k)=\sum_{i=0}^{k-1} \sum_{n=0}^{\infty} s^{\left\lfloor y_{n, i} \bar{x}_{n, i}\right\rfloor} \mathbb{P}\left(Y=y_{n, i} \bar{x}_{n, i}\right) \\
= & \sum_{i=0}^{k-1} \sum_{n=0}^{\infty} s^{\left\lfloor y_{n, i} \bar{x}_{n, i}\right\rfloor} \mathbb{P}(R=n k+i) \\
= & \sum_{i=0}^{k-1} \sum_{n=0}^{\infty} s^{\left\lfloor y_{n, i} G^{n k+i} \bar{c}_{i}\right\rfloor} \mathbb{P}(R=n k+i) \\
& \sum_{i=0}^{k-1} \mathbb{E}\left[s^{\left\lfloor\bar{c}_{i} h(R)\right\rfloor} I_{i}\right] .
\end{aligned}
$$

Se $\psi=\varphi_{Y}(\psi)$, então $\left.\sum_{i=0}^{k-1} \mathbb{E}\left[\psi\left\lfloor\bar{c}_{i} h(R)\right\rfloor I_{i}\right]\right)=\psi$. Pela construção, $\mathbb{P}\left(V^{C}\right) \geq$ $\psi$ donde $\mathbb{P}(V) \leq 1-\psi$. 
Teorema 4.2.2. Considere o processo Firework Discreto numa árvore periódica de graus $d_{1}+1, d_{2}+1, \cdots, d_{k}+1, d_{i} \geq 2$ para todo $i=1,2, \cdots k$. Suponha

$$
\sum_{i=0}^{k-1} c_{i} \mathbb{E}\left[G^{R} I_{i}\right]>1+\mathbb{P}(R=0) .
$$

Sejam $\rho$ e $\psi$ satisfazendo

$$
\begin{aligned}
& \sum_{i=0}^{k-1} \mathbb{E}\left[\rho^{c_{i} G^{R}} I_{i}\right]+(1-\rho) \mathbb{P}(R=0)=\rho \\
& \sum_{i=0}^{k-1} \mathbb{E}\left[\psi\left[\bar{c}_{i} h(R)\right\rfloor I_{i}\right]=\psi
\end{aligned}
$$

$\operatorname{com} h(R)=\frac{\left[d_{(1)}^{R}-1\right]}{d_{(1)}^{R-1}\left[d_{(1)}-1\right]} G^{R}$.

Então

$$
\begin{aligned}
& \mathbb{P}(V) \geq 1-\left(1-\rho^{1+\frac{1}{d_{(1)}}}\right) \mathbb{P}(R=0)-\sum_{i=0}^{k-1} \mathbb{E}\left(\rho^{c_{i}\left(1+\frac{1}{d_{(1)}}\right) G^{R}} I_{i}\right) e \\
& \left.\mathbb{P}(V) \leq 1-\sum_{i=0}^{k-1} \mathbb{E}\left(\psi^{\left\lfloor\bar{c}_{i}\left(1+\frac{1}{d_{(k)}}\right) h(R)\right\rfloor}\right\rfloor_{i}\right) .
\end{aligned}
$$

Prova do Teorema 4.2.2

Escreva

$$
V=V \bigcap\left[\bigcup_{k=0}^{\infty}\left\{R_{0}=k\right\}\right],
$$

onde $R_{0}$ é o alcance de explosão da partícula presente na raíz da árvore. Temos:

$$
\mathbb{P}(V)=\sum_{k=0}^{\infty} \mathbb{P}\left(V \mid R_{0}=k\right) \mathbb{P}\left(R_{0}=k\right)
$$

Lema 4.2.2.1.

$$
\mathbb{P}\left(V \mid R_{0}=n k+i\right) \geq 1-\rho^{\frac{x_{n, i}\left(d_{(1)}+1\right)}{d_{(1)}}} \quad i=1,2, \cdots
$$


Prova do Lema 4.2.2.1

Consideraremos a explosão emitida pela partícula na raíz. Se $R_{0}=n k+i$, temos pelo menos $\frac{x_{n, i}\left(d_{(1)}+1\right)}{d_{(1)}}$ partículas ativadas e que distam $\mathrm{k}$ da raíz. Considere $\frac{x_{n, i}\left(d_{(1)}+1\right)}{d_{(1)}}$ árvores cuja raíz possui uma partícula ativada nesta primeira explosão. Considere um processo Firework Discreto em cada uma dessas árvores. Nestes termos, defina os eventos: $V_{n k+i, j}$ : "Processo Firework Discreto relativo a j-ésima árvore sobrevive". Observe que cada uma destas árvores está imersa em uma árvore quase periódica de graus $d_{1}+1, d_{2}+1, \cdots, d_{k}+1, d_{i} \geq 2$ para todo $i=1,2, \cdots k$. Assim, $\mathbb{P}\left(V_{n k+i, j}\right) \in$ $[1-\rho, 1-\psi]$. Logo

$$
\begin{aligned}
& \mathbb{P}\left(V \mid R_{0}=n k+i\right) \geq \mathbb{P}\left(\bigcup_{j=1}^{\frac{x_{R_{0}\left(d_{(1)}+1\right)}}{d_{(1)}}} V_{R_{0}, j} \mid R_{0}=n k+i\right) \\
& =\mathbb{P}\left(\bigcup_{j=1}^{\frac{x_{n, i}\left(d_{(1)}+1\right)}{d_{(1)}}} V_{n k+i, j} \mid R_{0}=n k+i\right) \\
& =\mathbb{P}\left(\bigcup_{j=1}^{\frac{x_{n, i}\left(d_{(1)}+1\right)}{d_{(1)}}} V_{n k+i, j}\right) \\
& =1-\mathbb{P}\left(\bigcap_{j=1}^{\frac{x_{n, i}\left(d_{(1)}+1\right)}{d_{(1)}}}\left(V_{n k+i, j}\right)^{C}\right) \\
& \frac{x_{n, i}\left(d_{(1)}+1\right)}{d_{(1)}} \\
& =1-\prod_{j=1} \mathbb{P}\left(\left(V_{n k+i, j}\right)^{C}\right) \\
& =1-\prod_{j=1}^{\frac{x_{n, i}\left(d_{(1)}+1\right)}{d_{(1)}}} \rho=1-\rho^{\frac{x_{n, i}\left(d_{(1)}+1\right)}{d_{(1)}}} \text {. }
\end{aligned}
$$


Lema 4.2.2.2.

$$
\mathbb{P}\left(V \mid R_{0}=n k+i\right) \leq 1-\psi^{\left\lfloor\frac{y_{n, i} \bar{x}_{n, i}\left(d_{(k)}+1\right)}{d_{(k)}}\right\rfloor} \quad i=0,1, \cdots
$$

Prova do Lema 4.2.2.2

Se $R_{0}=n k+i$, temos menos que $\left\lfloor\frac{y_{n, i} \bar{x}_{n, i}\left(d_{(k)}+1\right)}{d_{(k)}}\right\rfloor$ partículas ativadas após a emissão de explosão da partícula na raíz. Considere $\left\lfloor\frac{y_{n, i} \bar{x}_{n, i}\left(d_{(k)}+1\right)}{d_{(k)}}\right\rfloor$ árvores cuja raíz possui uma partícula ativada nesta primeira explosão. Considere um processo Firework Discreto em cada uma dessas árvores. Quando uma destas árvores esta imersa em outra, consideraremos duas árvores distintas com dois processos Fireworks Discreto independentes. Nestes termos, defina os eventos: $V_{n k+i, j}$ : "Processo Firework Discreto relativo a j-ésima árvore sobrevive". Observe que cada uma destas árvores está imersa em uma árvore quase periódica de graus $d_{1}+1, d_{2}+1, \cdots, d_{k}+1, d_{i} \geq 2$ para todo $i=$ $1,2, \cdots k$. Assim, $\mathbb{P}\left(V_{n k+i, j}\right) \in[1-\rho, 1-\psi]$. Logo

$$
\begin{aligned}
& \mathbb{P}\left(V \mid R_{0}=n k+i\right) \leq \mathbb{P}\left(\bigcup_{j=1}^{\left\lfloor\frac{y_{R_{0}} \bar{x}_{R_{0}}(d(k)+1)}{d_{(k)}}\right\rfloor} V_{R_{0}, j} \mid R_{0}=n k+i\right) \\
& =\mathbb{P}\left(\bigcup_{j=1}^{\left\lfloor\frac{y_{n, i} \bar{x}_{n, i}\left(d_{(k)}+1\right)}{d_{(k)}}\right\rfloor} V_{n k+i, j} \mid R_{0}=n k+i\right) \\
& =\mathbb{P}\left(\begin{array}{l}
\left\lfloor\frac{y_{n, i} \bar{x}_{n, i}\left(d_{(k)}+1\right)}{d_{(k)}}\right\rfloor \\
\bigcup_{j=1} V_{n k+i, j}
\end{array}\right) \\
& =1-\mathbb{P}\left(\bigcap_{j=1}^{\left\lfloor\frac{y_{n, i} \bar{x}_{n, i}\left(d_{(k)}+1\right)}{d_{(k)}}\right\rfloor}\left(V_{n k+i, j}\right)^{C}\right) \\
& =1-\prod_{j=1}^{\left\lfloor\frac{y_{n, i} \bar{x}_{n, i}\left(d_{(k)}+1\right)}{d_{(k)}}\right\rfloor} \mathbb{P}\left(\left(V_{n k+i, j}\right)^{C}\right)
\end{aligned}
$$


60 CAPÍTULO 4. PROCESSO FIREWORK DISCRETO EM ÁRVORES

$$
\begin{aligned}
& =1-\prod_{j=1}^{\left\lfloor\frac{y_{n, i} \bar{x}_{n, i}\left(d_{(k)}+1\right)}{d_{(k)}}\right\rfloor} \psi \\
& =1-\psi^{\left\lfloor\frac{y_{n, i} \bar{x}_{n, i}\left(d_{(k)}+1\right)}{d_{(k)}}\right\rfloor} .
\end{aligned}
$$

Voltemos a prova do Teorema. Do Lema 4.2.2.1 segue

$$
\begin{aligned}
\mathbb{P}(V) & =\sum_{j=0}^{\infty} \mathbb{P}\left(V \mid R_{0}=j\right) \mathbb{P}\left(R_{0}=j\right)=\sum_{i=0}^{k-1} \sum_{n=0}^{\infty} \mathbb{P}\left(V \mid R_{0}=n k+i\right) \mathbb{P}\left(R_{0}=n k+i\right) \\
& =\sum_{n=1}^{\infty} \mathbb{P}\left(V \mid R_{0}=n k\right) \mathbb{P}\left(R_{0}=n k\right)+\sum_{i=1}^{k-1} \sum_{n=0}^{\infty} \mathbb{P}\left(V \mid R_{0}=n k+i\right) \mathbb{P}\left(R_{0}=n k+i\right) \\
& \geq \sum_{n=1}^{\infty}\left[1-\rho^{\frac{x_{n, 0}\left(d_{(1)}+1\right)}{d_{(1)}}}\right] \mathbb{P}(R=n k)+\sum_{i=1}^{k-1} \sum_{n=0}^{\infty}\left[1-\rho^{\frac{x_{n, i}\left(d_{(1)}+1\right)}{d_{(1)}}}\right] \mathbb{P}(R=n k+i) \\
& =1-\mathbb{P}(R=0)+\rho^{\frac{d_{(1)}+1}{d(1)}} \mathbb{P}(R=0)-\sum_{i=0}^{k-1} \sum_{n=0}^{\infty} \rho^{\frac{x_{n, i}\left(d_{(1)}+1\right)}{d_{(1)}}} \mathbb{P}(R=n k+i) \\
& =1-\left(1-\rho^{\frac{d_{(1)}+1}{d_{(1)}}}\right) \mathbb{P}(R=0)-\sum_{i=0}^{k-1} \sum_{n=0}^{\infty} \rho^{c_{i} \frac{\left(d_{(1)}+1\right)}{d_{(1)}} G^{n k+i}} \mathbb{P}(R=n k+i) \\
& =1-\left(1-\rho^{\frac{d_{(1)}+1}{d_{(1)}}}\right) \mathbb{P}(R=0)-\sum_{i=0}^{k-1} \mathbb{E}\left[\rho^{c_{i} \frac{\left(d_{(1)}+1\right)}{d_{(1)}} G^{R}} I_{i}\right] .
\end{aligned}
$$

Do Lema 4.2.2.2 segue

$$
\begin{aligned}
& \mathbb{P}(V)=\sum_{j=0}^{\infty} \mathbb{P}\left(V \mid R_{0}=j\right) \mathbb{P}\left(R_{0}=j\right) \\
& \leq \sum_{i=0}^{k-1} \sum_{n=0}^{\infty}\left[1-\psi^{\left\lfloor\frac{y_{n, i} \bar{x}_{n, i}\left(d_{(k)}+1\right)}{d_{(k)}}\right\rfloor}\right] \mathbb{P}(R=n k+i) \\
& =1-\sum_{i=0}^{k-1} \sum_{n=0}^{\infty}\left[\psi^{\left\lfloor\frac{y_{n, i} \bar{x}_{n, i}\left(d_{(k)}+1\right)}{d_{(k)}}\right\rfloor}\right] \mathbb{P}(R=n k+i)
\end{aligned}
$$

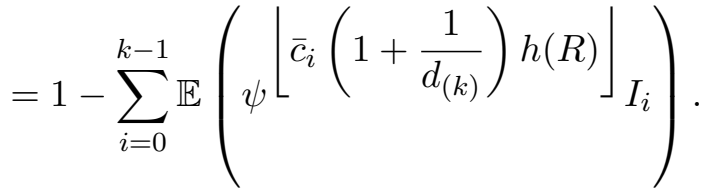


Exemplo 4.2.2.1. Seja um processo Firework Discreto na árvore 4-periódica com $d_{1}=2, d_{2}=3, d_{3}=4$ e $d_{4}=5$ tal que

$$
\mathbb{P}(R=j)=\left(\begin{array}{l}
4 \\
j
\end{array}\right)\left(\frac{1}{4}\right)^{j}\left(\frac{3}{4}\right)^{4-j}, j=0,1,2,3,4 .
$$

Temos $0,660963502 \leq \mathbb{P}(V) \leq 0,683159352$.

\section{3 Árvore Esfericamente Simétrica}

Uma árvore esfericamente simétrica é aquela onde vértices equidistantes da raiz têm o mesmo grau. Uma árvore deste tipo pode ser obtida a partir de uma seqüência de inteiros positivos $n_{1}, n_{2}, \cdots$ da seguinte forma. A raiz $O$ tem $n_{1}$ sucessores, cada um destes $n_{1}$ sucessores tem $n_{2}$ sucessores e assim por diante.

Considere o processo Firework Discreto na árvore esfericamente simétrica. Em [2], os autores encontram condições de sobrevivência para o caso onde os alcances têm distribuição geométrica.

Seja $u \leq v$, defina o evento $V_{u, v}$ : "Processo partindo de $u$ atinge $v$ ".

Fixado $n$, defina os conjuntos aleatórios

$$
\begin{aligned}
& X_{0}^{n}:=\{O\} \text { e para } j=1,2, \cdots \\
& X_{j}^{n}:=\bigcup_{u \in X_{j-1}^{n}}\left\{v \in \partial T_{n}^{u}: V_{u, v} \text { ocorre. }\right\}
\end{aligned}
$$

Agora, para todo $j=0,1,2, \cdots$ defina as variáveis aleatórias

$$
Z_{j}^{n}:=\left|X_{j}^{n}\right|
$$

Logo, para todo $n \geq 1$ fixado, $\left(Z_{j}^{n}\right)_{j \geq 0}$ é um processo de ramificação cuja sobrevivência implica na sobrevivência do processo Firework Discreto.

Lema 4.3.0.3. Para o processo $\left(Z_{j}^{n}\right)_{j \geq 0}$, o número médio de descendentes de um indivíduo da j-ésima geração satisfaz

$$
\mu_{j}:=\mu_{j}^{n}=M_{n}(v) \cdot \rho_{n},
$$

onde $|v|=j n$ e $\rho_{n}=\mathbb{P}\left(V_{u, w}\right)$, tal que $|u|+n=|w|$ e $u \leq w$. 
Prova do Lema 4.3.0.3

Fixados $j$ e $n$ :

$$
\partial T_{n}^{v}=\left\{u_{1}, u_{2}, \cdots, u_{M_{n}(v)}\right\}
$$

Temos

$$
Z_{j}^{n}=\sum_{i=1}^{M_{n}(v)} I_{\left\{V_{v, u_{i}}\right\}}
$$

Logo

$\mu_{j}^{n}=\mathbb{E}\left(Z_{j}^{n}\right)=\mathbb{E}\left(\sum_{i=1}^{M_{n}(v)} I_{\left\{V_{v, u_{i}}\right\}}\right)=\sum_{i=1}^{M_{n}(v)} \mathbb{E}\left(I_{\left\{V_{v, u_{i}}\right\}}\right)=\sum_{i=1}^{M_{n}(v)} \mathbb{P}\left(V_{v, u_{i}}\right)=M_{n}(v) \cdot \rho_{n}$.

Proposição 4.3.0.1. Numa árvore esfericamente simétrica, o processo Firework Discreto sobrevive com probabilidade positiva se

$$
\lim _{n \rightarrow \infty} \sqrt[n]{\rho_{n}}>e^{-\operatorname{dim} \inf \partial T}
$$

Prova da Proposição 4.3.0.1

Suponha $\operatorname{dim} \inf \partial T>0$. Logo, para todo $\alpha \in(0, \operatorname{dim} \inf \partial T)$ existe $N=N(\alpha)$ tal que para todo $n \geq N$

$$
\min _{v \in \mathcal{V}} \frac{1}{n} \ln M_{n}(v)>\alpha
$$

donde

$$
M_{n}(v) \geq e^{\alpha n} \text { para todo } v \in \mathcal{V} \text { e } n \geq N .
$$

Por outro lado, um processo de ramificação em meios variáveis é uniformemente supercrítico se

$$
\liminf _{j \rightarrow \infty} \mu_{j}>1 \text { (veja apêndice). }
$$

Do Lema 4.3.0.3 temos para $n \geq N$

$$
\liminf _{j \rightarrow \infty} \mu_{j} \geq e^{\alpha n} \rho_{n}=\left(e \sqrt[n]{\rho_{n}}\right)^{n}
$$

Além disso, observe que $\frac{Z_{j}^{n}}{\mathbb{E}\left(Z_{j}^{n}\right)} \leq \frac{1}{\rho_{n}}$. Logo, o processo sobrevive com probabilidade positiva se

$\lim _{n \rightarrow \infty} e \sqrt[n]{\rho_{n}}>1$, isto é, se $\lim _{n \rightarrow \infty} \sqrt[n]{\rho_{n}}>e^{-\alpha}$ (veja Teorema 5.4.1 no apêndice). 
Como isto vale para todo $\alpha \in(0, \operatorname{dim} \inf \partial T)$, a condição

$$
\lim _{n \rightarrow \infty} \sqrt[n]{\rho_{n}}>e^{-\operatorname{dim} \inf \partial T}
$$

garante sobrevivência com probabilidade positiva.

Corolário 4.3.0.1. Considere o processo Firework Discreto na árvore esfericamente simétrica com as variáveis aleatórias $R$ seguindo uma lei de potência, isto é,

$$
\mathbb{P}(R=k)=\frac{Z_{\alpha}}{(k+1)^{\alpha}} \text { para } k=0,1, \cdots,
$$

onde $Z_{\alpha}$ é uma constante de normalização e $\alpha>1$. O processo sobrevive com probabilidade positiva se

$$
\operatorname{dim} \inf \partial T>0 .
$$

Prova do Corolário 4.3.0.1

Observe que

$\rho_{n} \geq \mathbb{P}(R \geq n)=\sum_{k=n}^{\infty} \frac{Z_{\alpha}}{(k+1)^{\alpha}}=\sum_{j=n+1}^{\infty} \frac{Z_{\alpha}}{j^{\alpha}} \geq \int_{n+1}^{\infty} \frac{Z_{\alpha}}{x^{\alpha}} d x=\frac{Z_{\alpha}}{(\alpha-1)(n+1)^{\alpha-1}}$.

A desigualdade acima segue do teste da integral.

Agora observe que se dim inf $\partial T>0$, temos

$$
\lim _{n \rightarrow \infty} \sqrt[n]{\rho_{n}} \geq \lim _{n \rightarrow \infty} \sqrt[n]{\frac{Z_{\alpha}}{(\alpha-1)} \frac{1}{(n+1)^{\alpha-1}}}=1>e^{\operatorname{dim} \inf \partial T}
$$

A Proposição 4.3.0.1 nos garante o resultado desejado.

Teorema 4.3.1. O processo Firework Discreto na árvore esfericamente simétrica sobrevive com probabilidade positiva se

$$
\lim _{n \rightarrow \infty} \sqrt[n]{\prod_{i=0}^{n-1}\left[1-\prod_{j=0}^{i} \mathbb{P}(R<j+1)\right]}>e^{-\operatorname{dim} \inf \partial T .}
$$

Prova do Teorema 4.3.1

Considere o processo Firework simples sobre $\mathbb{Z}$. Neste, seja $V_{n}$ o evento : "Partícula no vértice $n$ é ativada". Note que

$$
\mathbb{P}\left(V_{n}\right)=\rho_{n} .
$$


Corolário 4.3.1.1. Seja o processo Firework Discreto na árvore esfericamente simétrica. Se $\mathbb{P}(R>k)=0$, então o processo sobrevive com probabilidade positiva se

$$
\prod_{j=1}^{k} \mathbb{P}(R<j)<1-e^{-\operatorname{dim} \inf \partial T} .
$$

Prova do Corolário 4.3.1.1

$$
\begin{aligned}
& \sqrt[n]{\prod_{i=0}^{n-1}\left[1-\prod_{j=0}^{i} \mathbb{P}(R<j+1)\right]}=\left[1-\prod_{j=1}^{k} \mathbb{P}(R<j)\right] \sqrt[n]{\frac{\prod_{i=0}^{k-1}\left[1-\prod_{j=0}^{i} \mathbb{P}(R<j+1)\right]}{\left(1-\prod_{j=1}^{k} \mathbb{P}(R<j)\right)^{k}}} \\
& \rightarrow 1-\prod_{j=1}^{k} \mathbb{P}(R<j), \text { quando } n \rightarrow \infty .
\end{aligned}
$$

Exemplo 4.3.1.1. Seja um processo Firework Discreto na árvore esfericamente simétrica tal que $\mathbb{P}(R=0)=1-p$ e $\mathbb{P}(R=1)=p$. O processo sobrevive com probabilidade positiva se

$$
p>e^{-\operatorname{dim} \inf \partial T} \text { isto é, se } \operatorname{dim} \inf \partial T>\ln \frac{1}{p} .
$$

Em particular, se considerarmos o processo numa árvore k-periódica de graus $d_{1}, d_{2}, \cdots, d_{k}$, o processo sobrevive com probabilidade positiva se

$$
G>\frac{1}{p}
$$

onde

$$
G=\sqrt[k]{\prod_{i=1}^{k} d_{i}}
$$

é a média geométrica dos valores $d_{1}, d_{2}, \cdots, d_{k}$.

Exemplo 4.3.1.2. Seja um processo Firework Discreto na árvore esfericamente simétrica tal que $\mathbb{P}(R=0)=p, \mathbb{P}(R=1)=q$ e $\mathbb{P}(R=2)=r$. O processo sobrevive com probabilidade positiva se

$$
\operatorname{dim} \inf \partial T>\ln \frac{1}{[1-p(p+q)]} .
$$




\section{Capítulo 5}

\section{Apêndice}

\subsection{Resultados básicos}

Lema 5.1.0.1. Desigualdade de $F K G$

Sejam $X$ e $Y$ duas variáveis aleatórias crescentes e limitadas em $\Omega$. Então

$$
\mathbb{E}(X Y) \geq \mathbb{E}(X) \mathbb{E}(Y)
$$

Em particular se $A$ e $B$ forem eventos crescentes, então

$$
\mathbb{P}(A \cap B) \geq \mathbb{P}(A) \mathbb{P}(B) .
$$

Prova do Lema 5.1.0.1

Ver [7] páginas 14 e 15.

Lema 5.1.0.2. Se $\left\{a_{k}\right\}$ é uma sequência de números reais em $(0,1)$ então

$$
\prod_{k=1}^{\infty}\left(1-a_{k}\right)>0 \Leftrightarrow \sum_{k=1}^{\infty} a_{k}<\infty .
$$

Prova do Lema 5.1.0.2

Usando $1-a_{k}<e^{-a_{k}}$ temos

$$
\prod_{k=1}^{\infty}\left(1-a_{k}\right) \leq \exp \left\{-\sum_{k=1}^{\infty} a_{k}\right\} \text {. }
$$

Logo

$$
\prod_{k=1}^{\infty}\left(1-a_{k}\right)=0 \text { se } \sum_{k=1}^{\infty} a_{k}=\infty .
$$


Por outro lado, para qualquer $\epsilon>0$ fixado, se $\sum_{k=1}^{\infty} a_{k}<\infty$ então $\sum_{k=n_{0}}^{\infty} a_{k}<\epsilon$ para algum $n_{0} \in \mathbb{N}$. Logo:

$$
\begin{aligned}
& \prod_{k=1}^{\infty}\left(1-a_{k}\right)=\prod_{k=1}^{n_{0}-1}\left(1-a_{k}\right) \prod_{k=n_{0}}^{\infty}\left(1-a_{k}\right) \geq \prod_{k=1}^{n_{0}-1}\left(1-a_{k}\right)\left[1-\sum_{k=n_{0}}^{\infty} a_{k}\right]> \\
& (1-\epsilon) \prod_{k=1}^{n_{0}-1}\left(1-a_{k}\right)>0 .
\end{aligned}
$$

Lema 5.1.0.3. Teste de Raabe

Dada uma série de termos positivos $a_{n}$. Seja

$$
L=\lim _{n \rightarrow \infty} n\left(\frac{a_{n}}{a_{n+1}}-1\right) .
$$

Se $L>1$ então $\sum_{n=1}^{\infty} a_{n}<\infty$.

Se $L<1$ então $\sum_{n=1}^{\infty} a_{n}=\infty$.

Se $L=1$ e existe $N$ tal que, $n>N \Rightarrow n\left(\frac{a_{n}}{a_{n+1}}-1\right)<1$ então $\sum_{n=1}^{\infty} a_{n}=\infty$.

Prova do Lema 5.1.0.3

Ver [1].

\subsection{Generalização do Lema de Borel-Cantelli}

O Lema de Borel-Cantelli é um importante resultado da teoria da probabilidade. Este resultado diz o seguinte: se $A_{1}, A_{2}, \cdots$ é uma sequência de eventos sobre um mesmo espaço de probabilidade e

$$
\sum_{n=1}^{\infty} \mathbb{P}\left(A_{n}\right)<\infty
$$


então

$$
\mathbb{P}\left(\limsup _{n \rightarrow \infty} A_{n}\right)=0
$$

se $A_{1}, A_{2}, \cdots$ é uma sequência de eventos independentes sobre um mesmo espaço de probabilidade e

$$
\sum_{n=1}^{\infty} \mathbb{P}\left(A_{n}\right)=\infty
$$

então

$$
\mathbb{P}\left(\limsup _{n \rightarrow \infty} A_{n}\right)=1
$$

Aqui

$$
\limsup _{n \rightarrow \infty} A_{n}=\bigcap_{n=1}^{\infty} \bigcup_{k=n}^{\infty} A_{k}
$$

é o evento $A_{n}$ infinitas vezes.

Nos últimos anos muito esforço foi devotado para enfraquecer a condição de independência. Dentre alguns resultados obtidos apresentamos o seguinte:

Teorema 5.2.1. Seja $A_{1}, A_{2}, \cdots$ uma sequência de eventos satisfazendo

$$
\sum_{n=1}^{\infty} \mathbb{P}\left(A_{n}\right)=\infty
$$

$e$

$$
\mathbb{P}\left(A_{k} \cap A_{j}\right) \leq \mathbb{P}\left(A_{k}\right) \mathbb{P}\left(A_{j}\right)
$$

para todo $k$ e $j$ suficientemente grande $e k \neq j$. Então

$$
\mathbb{P}\left(\limsup _{n \rightarrow \infty} A_{n}\right)=1 \text {. }
$$

Prova do Teorema 5.2.1

A prova deste resultado pode ser encontrada em [14].

\subsection{Processos de Galton-Watson}

Um processo de Galton-Watson ( processo de ramificação ) é uma Cadeia de Markov $\left\{Z_{n}\right\}_{n \geq 0}$ sobre os inteiros não-negativos. Sua função de transição 
é especificada por uma lei de probabilidade $\left\{p_{k}, k=0,1, \cdots\right\}, p_{k} \geq 0$, $\sum p_{k}=1 \mathrm{com}$

$$
P_{i j}=\mathbb{P}\left[Z_{n+1}=j \mid Z_{n}=i\right]= \begin{cases}p_{j}^{* i}, & \text { se } i \geq 1, j \geq 0 \\ \delta_{0 j}, & \text { se } i=0, j \geq 0\end{cases}
$$

onde

$$
p_{j}^{* i}=\sum_{j_{1}+j_{2}+\cdots+j_{i}=j} p_{j_{1}} p_{j_{2}} \cdots p_{j_{i}}
$$

Podemos escrever

$$
Z_{n+1}=\sum_{i=1}^{Z_{n}} X_{i}^{n}
$$

onde as variáveis aleatórias $X_{i}^{n}$ são independentes e além disso identicamente distribuídas como uma variável aleatória $X$. Podemos pensar esse processo como a quantidade de descendentes de uma população ao longo de gerações. Assim, se $Z_{0}=1$ então temos na geração inicial um único indivíduo. Se $Z_{1}=k$, então o individuo inicial teve $k$ descendentes e nesse caso, a segunda geração terá $X_{1}+X_{2}+\cdots+X_{k}$ descendentes e assim por diante. Nessa vertente é bom ressaltar que a distribuição de probabilidade do número de descendentes de um indivíduo é a mesma para todos os indivíduos.

Dizemos que o processo de Galton-watson se extingue se $Z_{n}=0$ para algum $n$. Seja

$$
q=\mathbb{P}\left(\lim _{n \rightarrow \infty} Z_{n}=0\right) .
$$

Teorema 5.3.1. Para um processo de ramificação $\left\{Z_{n}\right\}_{n \geq 0}$ temos

1. $q=1$ se $\mathbb{E}(X)<1$,

2. $q<1$ se $\mathbb{E}(X)>1$,

3. $q<1$ se $\mathbb{E}(X)=1$ e $\mathbb{P}(X=1)<1$.

Além disso, se $\varphi_{X}$ é a função geradora de probabilidade de $X$, isto é,

$$
\varphi_{X}(s):=\mathbb{E}\left(s^{X}\right)
$$

então a probabilidade de extinção $q$ é a menor solução em $[0,1]$ de $q=\varphi_{X}(q)$.

Prova do Teorema 5.3.1

A prova deste teorema bem como teoria e outros resultados importantes sobre processos de Galton-Watson podem ser encontrados em [8] ou [13]. 


\subsection{PROCESSOS DE GALTON-WATSON EM MEIOS ALEATÓRIOS69}

\subsection{Processos de Galton-Watson em Meios Aleatórios}

Um processo de Galton-Watson em meio aleatório é uma generalização do processo de Galton-Watson onde a distribuição do número de descendentes depende do número da geração. Assim, se $Z_{j}$ é o número de indivíduos na j-ésima geração então

$$
Z_{j+1}=\sum_{i=1}^{Z_{j}} X_{j, i}, j \geq 0
$$

onde $\left(X_{j, i}\right)_{i}$ são cópias independentes e identicamente distribuídas de $X_{j}$. O índice $j$ indica que a distribuição de $X_{j}$ depende do número $j$ que indica a geração.

Considere um processo de Galton-Watson em meio aleatório começando com um único indivíduo, isto é, $Z_{0}=1$. Suponha que o número médio de descendentes é finito e positivo, ou seja, $0<\mathbb{E}\left(X_{j}\right)<\infty$, para todo $j \geq 0$.

Seja $\mu_{j}:=\mathbb{E}\left(X_{j}\right)$ e $m_{j}=\mathbb{E}\left(Z_{j}\right)$. Temos $m_{0}=1 \mathrm{e}$

$$
m_{j}=\prod_{i=0}^{j-1} \mu_{i}, \text { para } j \geq 1
$$

De fato,

$$
\mathbb{E}\left(Z_{j} \mid Z_{j-1}=k\right)=\mathbb{E}\left(\sum_{i=1}^{k} X_{j-1, i} \mid Z_{j-1}=k\right)=\sum_{i=1}^{k} \mathbb{E}\left(X_{j-1, i}\right)=k \mu_{j-1}
$$

donde $m_{j}=\mathbb{E}\left(Z_{j}\right)=\mu_{j-1} \mathbb{E}\left(Z_{j-1}\right)$.

Para obter um resultado para a probabilidade de extinção deste processo vamos impor uma condição sobre o crescimento das médias. Para isto, dizemos que o processo é uniformemente supercrítico se existem constantes $a>0$ e $c>1$ tais que

$$
\prod_{k=i}^{j+i-1} \mu_{k} \geq a c^{j}
$$

para todo $i \geq 0$ e $j \geq 0$. Isto vale, por exemplo, sempre que

$$
\liminf _{j \rightarrow \infty} \mu_{j}>c>1 \text {. }
$$


Por outro lado, é preciso controlar o comportamento caudal das variáveis aleatórias $X_{j}$. Nós dizemos que a sequência $X_{j}$ é dominada pela variável aleatória $X$ se para cada $j$,

$$
\mathbb{P}\left(X_{j}>x\right) \leq \mathbb{P}(X>x) \text {, para todo } x .
$$

Por fim, denotamos a probabilidade de extinção do processo por $q$, ou seja,

$$
q=\mathbb{P}\left(\lim _{j \rightarrow \infty} Z_{j}=0\right) .
$$

Teorema 5.4.1. Se o processo é uniformemente supercrítico e a sequência $\frac{X_{j}}{\mu_{j}}$ é dominada por uma variável $X$ com $\mathbb{E}(X)<\infty$ então $q \leq \eta<1$, com $\eta$ dependendo somente de $a, c, e X$.

Prova do Teorema 5.4.1

A prova deste teorema bem como teoria e outros resultados importantes sobre processos de Galton-Watson em meios aleatórios podem ser encontrados em $[6]$. 


\section{Referências Bibliográficas}

[1] B. Ram, V.K. Srinivasan (1978). Remarks on Raabe's test in infinite series. INT. J. MATH. EDUC. SCI. TECHNOL., 1978, vol. 9, Número $3,361-363$.

[2] E.Lebensztayn, P.M. Rodriguez (2008).The disk-percolation model on graphs. Statistics \& Probability Letters, 2008, vol. 78, issue 14, pages 2130-2136.

[3] F.P. Machado, E. Lebensztayn, M. Z. Martinez (2005) Self avoiding random walks on homogeneous trees. Markov Processes and Related Fields, v. 12 , p. $735-745,2006$.

[4] F.P. Machado, E. Lebensztayn, M. Z. Martinez (2008) Random walks systems with killing em $\mathbb{Z}$. Stochastics and Stochastics Reports, v. 78, p. 1-15, 2007.

[5] G.Grimmett (1999). Percolation, 2 edição. Springer-Verlag, New York.

[6] J.C. D‘Souza, J.D. Biggins (1992). The supercritical Galton-Watson process in varying environments. Stochastic Process. Appl. 42(1), 39-47.

[7] L. R. G. Fontes. Notas em Percolação. IMPA, CNPq, 1996.

[8] R. Durret, Probability: theory and examples, (2nd edn.), Duxburry, Belmont. Calif.

[9] O.S.M. Alves, F.P. Machado, E. Lebensztayn, M. Z. Martinez (2006) Random walk systems on complete graphs. Bulletin of the Brazilian Mathematical Society, v. 37, p. 571-580, 2006.

[10] R. Lyons, Y. Peres (2005). Probability on trees and networks. Disponível em http://php.indiana.edu/ rdlyons/prbtree/book.pdf. 
[11] Siva Athreya, Rahul Roy, and Anish Sarkar (2004) On the coverage of space by random sets. Advances in Applied Probability. Volume 36, Number 1 (2004), 1-18.

[12] S.P.Meyn, R.L. Tweedie (1993) Markov chains and Stochastic Stability.

[13] S.H. Ross, Stochastic Processes, Wiley Series in Probability and Mathematical Statistics, 1996.

[14] V.V.Petrov (2002). A note on the Borel-Cantelli Lemma. Statistics \& Probability Letters 58 283-286.

[15] W. Feller, An Introduction to Probability Theory and its Applications, Wiley, New York,1966. 